\title{
Hybrid Rydberg quantum gate for quantum network
}

\author{
Yubao $\mathrm{Liu} \odot, \mathrm{Lin} \mathrm{Li} \odot,{ }^{*}$ and Yiqiu $\mathrm{Ma}^{\dagger}$ \\ Center for Gravitational Experiment, Institute for Quantum Science, Hubei Key Laboratory of Gravitation and Quantum Physics, \\ School of Physics, Huazhong University of Science and Technology, Wuhan, 430074, People's Republic of China
}

(Received 25 May 2021; accepted 30 November 2021; published 3 January 2022)

\begin{abstract}
The high-fidelity storage, distribution, and processing of quantum information prefers qubits with different physical properties. Thus hybrid quantum gates interfacing different types of qubits are essential for the realization of complex quantum network structures. A Rydberg-atom-based physical quantum controlled-Z (CZ) gate is proposed to hybridly process the polarization-encoded single-photon optical qubit and the "Schrödinger cat" microwave qubit. The degradation of the fidelity under the influence of various noise channels, such as microwave cavity loss, spontaneous emission of atom states, and nonadiabaticity effect, etc., has been analyzed through detailed theoretical analysis by deriving the input-output relation of qubit fields. The feasibility and the challenges of the protocol within current technology are also discussed by analyzing the possible experimental parameter settings. Our work opens prospects for future large-scale quantum architecture based on hybrid quantum modules.
\end{abstract}

DOI: 10.1103/PhysRevResearch.4.013008

\section{INTRODUCTION}

A robust and functional quantum network [1,2] requires the simultaneous achievement of both high-fidelity local quantum operation and efficient distribution of quantum information between remote quantum nodes [3,4]. The combination of these two capabilities would enable many important applications, such as long distance quantum communication [5-9], distributed quantum computation [10-14], and quantum metrology [15-20]. These two capabilities have been separately demonstrated in various experiments [21,22], but it remains challenging to combine them into a single physical system. For example, microwave (MW) superconducting qubits feature high fidelity [23,24] but suffer severe losses and decoherence during propagation [25]. On the other hand, optical photons are ideal qubits for long-distance quantum state distribution [22], but fault-tolerant quantum gates with optical photons remain elusive [26,27]. Naturally, a practical quantum network would largely benefit from a hybrid platform [28-39] which bridges the gap between optical and MW qubits.

However, achieving such a hybrid optical-MW quantum gate remains an unmet challenge, since it demands a coherent interaction between optical and MW photons. The frequencies of optical and MW photonic qubits differ by more than four orders of magnitudes, making photonic-interference-based protocols inapplicable. Alternatively, it is possible to employ a

\footnotetext{
*li_lin@ hust.edu.cn

†myqphy@hust.edu.cn
}

Published by the American Physical Society under the terms of the Creative Commons Attribution 4.0 International license. Further distribution of this work must maintain attribution to the author(s) and the published article's title, journal citation, and DOI. quantum-nonlinear medium [40] with efficient matter-photon coupling to facilitate the photon-photon interaction [41-48], while the difficulty lies in designing a physical system that can interacts strongly and coherently with both MW and optical qubits at the single quanta level.

To tackle this longstanding problem, we propose a protocol for the realization of high-fidelity quantum controlled-Z (CZ) gate between optical and MW photons mediated by Rydberg atoms [49-51] in a MW cavity. Two Rydberg levels resonant with the cavity are employed to interface with the MW qubit [52-55], while the optical qubit interacts with the atoms via a polarization-dependent electromagnetic induced transparency (EIT) photon storage scheme [42,56-60]. The system dynamics and the gate fidelity are carefully analyzed with realistic experimental parameters. The result shows that a high-fidelity hybrid quantum logic gate can be achieved with our protocol [42,61]. Our work opens avenues for future quantum network architectures connecting distant quantum nodes.

\section{PROTOCOL}

As illustrated in Fig. 1, our quantum medium is an ensemble of Rubidium ( $\mathrm{Rb}$ ) atoms in a MW cavity resonant with the transition between Rydberg states $\left|r_{1}\right\rangle$ and $\left|r_{2}\right\rangle$. The optical qubit employs right/left circular polarization encoding $\left(|1\rangle_{o}^{R / L}\right)$, while the MW qubit is encoded in the even/odd parity of the Schrödinger's cat state $\mid$ even/odd $\rangle_{\mathrm{m}} \propto|\alpha\rangle \pm|-\alpha\rangle$. The gate protocol starts with the EIT storage of the optical qubit, in which the $|1\rangle_{o}^{R}\left(|1\rangle_{o}^{L}\right)$ qubit state is stored in the Rydberg state $\left|r_{1}\right\rangle$ (ground state $\left|g^{\prime}\right\rangle$ ) using ladder $(\Lambda)$ level schemes, respectively. Next, a MW qubit resonant with the $\left|r_{1}\right\rangle-\left|r_{2}\right\rangle$ transition is reflected off the atom cavity system in the strong-coupling regime, such that the population in Rydberg state $\left|r_{1}\right\rangle$ blocks the cavity transmission. As a result, 


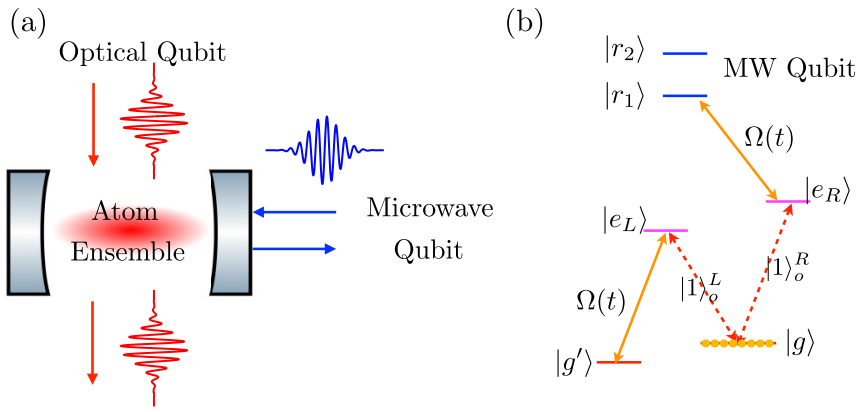

FIG. 1. Schematic setup: (a) an ensemble of $\mathrm{Rb}$ atoms trapped in a microwave (MW) cavity and (b) the atomic levels used in the hybrid quantum CZ gate. The atoms are initially populated on $|g\rangle$.

when the optical qubit is in state $|1\rangle_{o}^{R}$ (stored in $\left|r_{1}\right\rangle$ ), the MW qubit reflects from the input mirror and acquires no additional phase. With the optical qubit in state $|1\rangle_{o}^{L}$ (stored in $\left|g^{\prime}\right\rangle$ ), the MW qubit enters the empty cavity so the $\mid$ odd $\rangle_{\mathrm{m}}$ state acquires a $\pi$ phase shift while the $\mid$ even $\rangle_{\mathrm{m}}$ state remains unaffected. The above controlled phase shift mechanism establishes our CZ gate, with the truth table shown in Table I and Fig. 2.

Different from the previous optical photon-photon gate, which has stringent requirements on the interactions between two Rydberg polaritons, our protocol is built on the robust Rydberg cavity-quantum electrodynamics (cQED) and features innate error resilience, which does not require a Rydberg blockade and has modest demands on experimental parameters. Our protocol has the following features: (1) benefiting from the rich Rydberg level structures, our protocol is applicable to MW qubit at various frequency bands; (2) by employing collective atomic encoding for optical qubit storage, the quantum information is stored redundantly in the atomic spin wave, offering robustness against particle loss; and (3) Rydberg states with low quantum principal number can be used to make the gate less sensitive to stray electric fields.

\section{THEORETICAL MODEL AND PHYSICAL REALIZATIONS}

We now set up a theoretical model for our scheme. The Hamiltonian for the free-propagating electromagnetic (EM) field (e.g., along $+z$ direction) is given by

$$
\hat{H}_{\mathrm{EM}}=\frac{i c \hbar}{2 L_{a}} \int_{-\infty}^{+\infty} \mathrm{d} z\left[\frac{\partial \hat{e}^{\dagger}}{\partial z} \hat{e}-\frac{\partial \hat{e}}{\partial z} \hat{e}^{\dagger}\right],
$$

TABLE I. Truth table of the proposed hybrid quantum $\mathrm{CZ}$ gate. The loss in the EIT storage process contributes to the inefficiency, and the fidelity here is calculated considering the postselection of the output optical single-photon qubit.

\begin{tabular}{lccc}
\hline \hline Input state & Output state & Efficiency & Fidelity \\
\hline$|1\rangle_{o}^{L} \otimes \mid$ even $\rangle_{m}$ & $+|1\rangle_{o}^{L} \otimes \mid$ even $\rangle_{m}$ & 0.74 & 0.923 \\
$|1\rangle_{o}^{L} \otimes \mid$ odd $\rangle_{m}$ & $-|1\rangle_{o}^{L} \otimes \mid$ odd $\rangle_{m}$ & 0.74 & 0.923 \\
$|1\rangle_{o}^{R} \otimes \mid$ even $\rangle_{m}$ & $+|1\rangle_{o}^{R} \otimes \mid$ even $\rangle_{m}$ & 0.45 & 0.969 \\
$|1\rangle_{o}^{R} \otimes \mid$ odd $\rangle_{m}$ & $+|1\rangle_{o}^{R} \otimes \mid$ odd $\rangle_{m}$ & 0.45 & 0.967 \\
\hline \hline
\end{tabular}

(a) Rydberg states

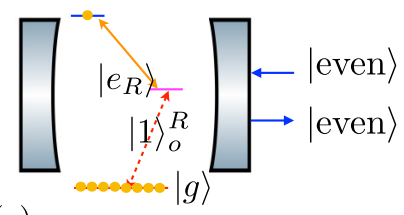

(c)

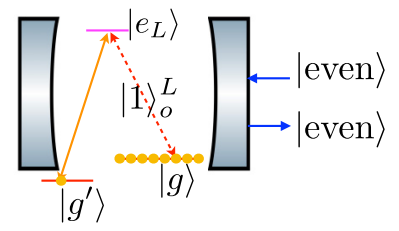

(d)

(b) Rydberg states

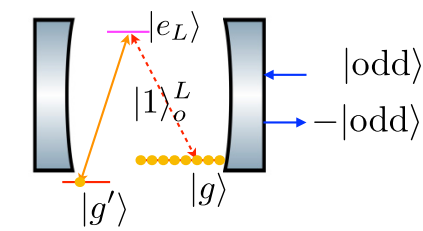

FIG. 2. Physical processes correspond to different qubit inputs of the quantum hybrid $\mathrm{CZ}$ gate, with the truth table in Table I.

where the $\hat{e}^{(\dagger)}$ is the annihilation (creation) operator of the EM slowly varying amplitude fields, and $c$ and $L_{a}$ are the vacuum light speed and the length of atomic medium, respectively. In the following, $\hat{e}$ is written as $\hat{\epsilon} / \hat{b}$ for the optical/MW qubit, respectively.

The atomic ensemble and the atom-light interaction are described by the following Hamiltonian [57-59]:

$$
\begin{aligned}
\hat{H}_{\text {atom }} & =-\hbar \sum_{j=1}^{N}\left(\omega_{a} \hat{\sigma}_{a a}^{j}+\omega_{c} \hat{\sigma}_{c c}^{j}\right), \\
\hat{H}_{\text {int }} & =-\hbar \sum_{j=1}^{N}\left[\Omega\left(z_{j}, t\right) e^{-i v_{\Omega} t+i k_{\Omega} z_{j}} \hat{\sigma}_{a c}^{j}+g \hat{\varepsilon} \hat{\sigma}_{b c}^{j}\right]+\text { H.c. },
\end{aligned}
$$

where $\hat{H}_{\text {atom }}$ describes an atom with energy levels $|b\rangle-$ $\left|a_{L / R}\right\rangle-|c\rangle, \hat{H}_{\text {int }}$ describes the interactions in the two EIT systems $\left(|g\rangle-\left|e_{L}\right\rangle-\left|g^{\prime}\right\rangle\right.$ and $\left.|g\rangle-\left|e_{R}\right\rangle-\left|r_{1}\right\rangle\right), \Omega_{R / L}\left(z_{j}, t\right)$ are the control fields, $g$ is the single-photon Rabi frequency, and $\hat{\epsilon}$ is the annihilation operator of the slowly varying amplitude fields for the optical qubit.

The interaction between the MW qubit and the cQED system is $[62,63]$

$$
\hat{H}_{\mathrm{CQED}}=\hbar g_{m} \hat{a}_{c} \hat{\sigma}_{r_{2} r_{1}}+\hbar \sqrt{\kappa} \hat{a}_{c} \hat{b}^{\dagger}(0)+\text { H.c. },
$$

where the first term describes the interaction between the MW cavity mode $\hat{a}_{c}$ and the two Rydberg states $\left|r_{1}\right\rangle,\left|r_{2}\right\rangle$ with single-photon Rabi frequency $g_{m}$, and the second term represents the MW qubit operator $\hat{b}$ interacting with the MW cavity mode with strength $\sqrt{\kappa}$ [64].

The system will be affected by the noisy environment, such as the vacuum field, which induces spontaneous emission, the collision of atoms, and cavity loss, etc. We denote these effects by $\gamma_{\mu \nu}$, which is the decoherence rate from $|\nu\rangle$ to $|\mu\rangle(\mu, \nu$ are among $\left.\left|g^{\prime}\right\rangle,|g\rangle,\left|e_{R}\right\rangle,\left|e_{L}\right\rangle\right), \gamma_{s}$ as the decay rate of the Rydberg state, and $\kappa_{s}$ the MW cavity loss rate. These factors will bring decoherence to the quantum state and affect the gate fidelity.

For later analysis, we consider the following example physical realization (Table II). The initial $\mathrm{Rb}$ atomic state is prepared in $|g\rangle=\left|5 S_{1 / 2}, F=m_{F}=2\right\rangle$, with its neighboring ground state $\left|g^{\prime}\right\rangle=\left|5 S_{1 / 2}, F=m_{F}=1\right\rangle$, and the intermediate quantum states $\left|e_{L}\right\rangle=\left|5 P_{3 / 2}, F=2, m_{F}=1\right\rangle$, and 
TABLE II. Sample parameters for our hybrid quantum gate protocol.

\begin{tabular}{lcc}
\hline \hline Rydberg state 1 & $\left|r_{1}\right\rangle$ & $\left|69 S_{1 / 2}, F=m_{F}=2\right\rangle$ \\
Rydberg state 2 & $\left|r_{2}\right\rangle$ & $\left|69 P_{3 / 2}, F=m_{F}=3\right\rangle$ \\
cQED coupling strength & $g_{m}$ & $2 \pi \times 2.723 \mathrm{MHz}$ \\
Decay rate of Rydberg state 1 & $\gamma_{r_{1} g}$ & $2 \pi \times 3.50 \mathrm{kHz}$ \\
Decay rate between & $\gamma_{r_{2} r_{1}}$ & $2 \pi \times 4.78 \mathrm{kHz}$ \\
$\quad$ Rydberg states & $\kappa$ & $2 \pi \times 2 \mathrm{MHz}$ \\
MW cavity bandwidth & $T_{0}$ & $0.5 \mu \mathrm{s}$ \\
Optical pulse duration time & $T_{\mathrm{EIT}}$ & $16 \mu \mathrm{s}$ \\
EIT storage time & $\gamma_{e g}$ & $2 \pi \times 3 \mathrm{MHz}$ \\
Decay rate of excited state & $w$ & $8 \mu m$ \\
Optical cross-sectional diameter & $w_{c}$ & $25 \mu m$ \\
Superconducting circuit & & $8 \times 10^{11} \mathrm{~cm}-3$ \\
$\quad$ electrode distance & $\varrho$ & $0.4 \mathrm{~mm}$ \\
Atom number density & $L_{a}$ & $2 \pi \times 0.012 \mathrm{MHz}$ \\
Length of atom ensemble & $g_{L}$ & \\
Vacuum Rabi frequency & & \\
$\quad$ for left-polarized optical field & & $g_{R}$ \\
$\quad$ Vacuum Rabi frequency & & $2 \pi \times 0.029 \mathrm{MHz}$ \\
$\quad$ for right-polarized optical field & & \\
\hline \hline
\end{tabular}

$\left|e_{R}\right\rangle=\left|5 P_{3 / 2}, F=m_{F}=3\right\rangle$. Besides, the Rydberg states are chosen to be $\left|r_{1}\right\rangle=\left|69 S_{1 / 2}, F=m_{F}=2\right\rangle$ and $\left|r_{2}\right\rangle=$ $\left|69 P_{3 / 2}, F=3, m_{F}=3\right\rangle$.

For the EIT storage of optical qubits: (1) The atomic ensemble has length $L_{a}=0.4 \mathrm{~mm}$ with density $\rho \sim 8 \times 10^{11} \mathrm{~cm}^{-3}$ and interaction cross-sectional diameter $w=8 \mu \mathrm{m}$. The single-photon Rabi frequencies for the $\left|e_{R}\right\rangle-|g\rangle$ and $\left|e_{L}\right\rangle-|g\rangle$ transitions are $g_{R}=2 \pi \times 29 \mathrm{kHz}$ and $g_{L}=2 \pi \times 12 \mathrm{kHz}$, respectively. (2) The two control field Rabi frequencies for the EIT processes are $\Omega_{R / L}(t)=$ $\Omega_{0}(2+\tanh [20(t-18 \mu s]-\tanh [20(t-2 \mu s)]) / 2, \quad$ where $\Omega_{0}=2 \pi \times 30 \mathrm{MHz}$, and the storage time is $16 \mu \mathrm{s}$. (3) The decay rates of atom states are $\gamma_{e g}=2 \pi \times 3 \mathrm{MHz}$, $\gamma_{r_{1} g}=2 \pi \times 3.5 \mathrm{kHz}$ [65], and $\gamma_{g^{\prime} g}=2 \pi \times 16 \mathrm{~Hz}$ [66]. This small $\gamma_{r_{1} g}$ requires the temperature of the atomic ensemble below $\sim 0.2 \mu \mathrm{K}$.

For the MW cQED system: the cavity has a quality factor $\sim 10^{4}$, the Rydberg state $\left|r_{2}\right\rangle$ has decay rate $\gamma_{s} \sim 2 \pi \times$ $4.78 \mathrm{kHz}$ [67], and the single-photon Rabi frequency is $g_{m}=$ $2 \pi \times 2.72 \mathrm{MHz}$ [68]. Such a strong $g_{m}$ demands a large dipole moment and a small interaction volume, which indicates a possible realization by combining Rydberg atoms with a superconducting circuit [34,39].

\section{FIELD OPERATOR EVOLUTION}

The above Hamiltonians can be solved under the adiabatic conditions (we leave a detailed derivation in Appendix A). Namely, the switching of the control field is slow enough to avoid the transition to bright states. The resultant Heisenberg equations of motion for describing the optical qubit evolution is given by

$$
\begin{aligned}
\left(\frac{\partial}{\partial t}+v \frac{\partial}{\partial z}\right) \hat{\varepsilon}(z, t)= & A \hat{\varepsilon}(z, t)+c^{2}(1-\eta)^{2} C \frac{\partial^{2}}{\partial z^{2}} \hat{\varepsilon}(z, t) \\
& +c^{3}(1-\eta)^{3} D \frac{\partial^{3}}{\partial z^{3}} \hat{\varepsilon}(z, t),
\end{aligned}
$$

where the $A>0 /<0$ represents the gain/attenuation of $\hat{\epsilon}$, corresponds to the retrieving/writing of the optical qubit, respectively. Coefficients $C$ and $D$ describe the qubit pulse spreading due to the limited EIT window and the higher-order distortion of the qubit wave form. These coefficients can be written as

$$
\begin{aligned}
& A=\eta\left[\frac{1}{\Omega} \frac{\partial \Omega}{\partial t}-\gamma_{b c}\right], \quad D=\frac{\eta}{|\Omega|^{2}}, \\
& C=\frac{\eta}{|\Omega|^{2}}\left[\left(2 \gamma_{b c}+\gamma_{b a}\right)-2\left(\frac{1}{\Omega^{*}} \frac{\partial \Omega^{*}}{\partial t}+\frac{2}{\Omega} \frac{\partial \Omega}{\partial t}\right)\right],
\end{aligned}
$$

in which $\eta=g^{2} N /\left(g^{2} N+|\Omega|^{2}\right)$ with $N$ the total number of atoms and $\Omega$ is the Rabi frequency of the control fields. The $v=c(1-\eta)$ is the qubit propagation velocity in the EIT medium, which corresponds to the slow light effect.

Solving the above equation leads to the input-output relation of the optical qubit field in the $k$ domain:

$$
\begin{aligned}
\hat{\varepsilon}(k, T)= & C_{1}^{o i}(k, T) \hat{\varepsilon}(k, 0)+C_{2}^{o i}(k, T) \hat{n}(k), \\
\text { with } \quad C_{1}^{o i}(k)= & \exp \left\{-i \int_{0}^{T} \mathrm{~d} t\left[k v-k^{3} c^{3} D(1-\eta)^{3}\right]\right\} \\
& \times \exp \left\{\int_{0}^{T} \mathrm{~d} t\left[A-k^{2} c^{2}(1-\eta)^{2} C\right]\right\},
\end{aligned}
$$

where $\hat{n}$ is the environmental noise operator, and the coefficients satisfy $\left|C_{1}^{o i}\right|^{2}+\left|C_{2}^{o i}\right|^{2}=1$ according to Caves's phenomenological theory of the quantum amplifier [69]. The optical qubit takes time $T$ to finish its full writing and retrieving process, and $i$ represents left/right (L/R) polarizations. The second exponential factor describes gain/attenuation of $\hat{\epsilon}$. The above formula also captures various loss channels, for example, the loss due to nonadiabaticity (nonzero $d \Omega / \Omega d t$ ) and the dissipation of various energy levels $\left(\gamma_{b c}, \gamma_{b a}\right)$.

For the $\mathrm{cQED}$ process, the equations of motion in the frequency domain are

$$
\begin{aligned}
\left(-i \omega+\gamma_{s}\right) \hat{\sigma}_{r_{1} r_{2}}(\omega)= & -i g_{m} \hat{a}_{c}(\omega)+\sqrt{2 \gamma_{s}} \hat{n}_{\text {en }}(\omega), \\
\left(-i \omega+\frac{\kappa+\kappa_{s}}{2}\right) \hat{a}_{c}(\omega)= & -i g_{m} \hat{\sigma}_{r_{1} r_{2}}(\omega)-\sqrt{\kappa} \hat{a}_{\text {in }}(\omega) \\
& -\sqrt{\kappa_{s}} \hat{n}(\omega),
\end{aligned}
$$

in deriving which we have used the linear approximation $\left\langle\hat{\sigma}_{z}\right\rangle \approx-1 / 2$. Solving these equations leads to the evolution of the MW qubit field:

$$
\hat{b}_{\text {out }}(\omega)=C_{1}(\omega) \hat{b}_{\text {in }}(\omega)+C_{2}(\omega) \hat{n}_{\text {in }}(\omega),
$$

where $\hat{n}_{\text {in }}$ represents all possible noise contributions (includes the cavity loss and Rydberg state decay) and

$$
\begin{aligned}
& C_{1}(\omega)=\frac{i \omega+g_{m}^{2} /\left(i \omega-\gamma_{s}\right)-\left(\kappa_{s}-\kappa\right) / 2}{i \omega+g_{m}^{2} /\left(i \omega-\gamma_{s}\right)-\left(\kappa_{s}+\kappa\right) / 2}, \\
& C_{2}(\omega)=\frac{i \omega-\gamma_{\mathrm{eff}}}{i \omega-\gamma_{s}} \frac{\sqrt{\kappa \kappa_{s}}}{i \omega+g_{m}^{2} /\left(i \omega-\gamma_{s}\right)-\left(\kappa_{s}+\kappa\right) / 2}, \\
& \left|C_{1}(\omega)\right|^{2}+\left|C_{2}(\omega)\right|^{2}=1,
\end{aligned}
$$

with $\gamma_{\mathrm{eff}}=\sqrt{\gamma_{s}^{2}+2 g_{m}^{2} \gamma_{s} / \kappa_{s}}$. In the strong-coupling region $g_{m}^{2} \gg \gamma \kappa$, the output field has a zero phase shift compared to the input field. However, in case the Rydberg state is 


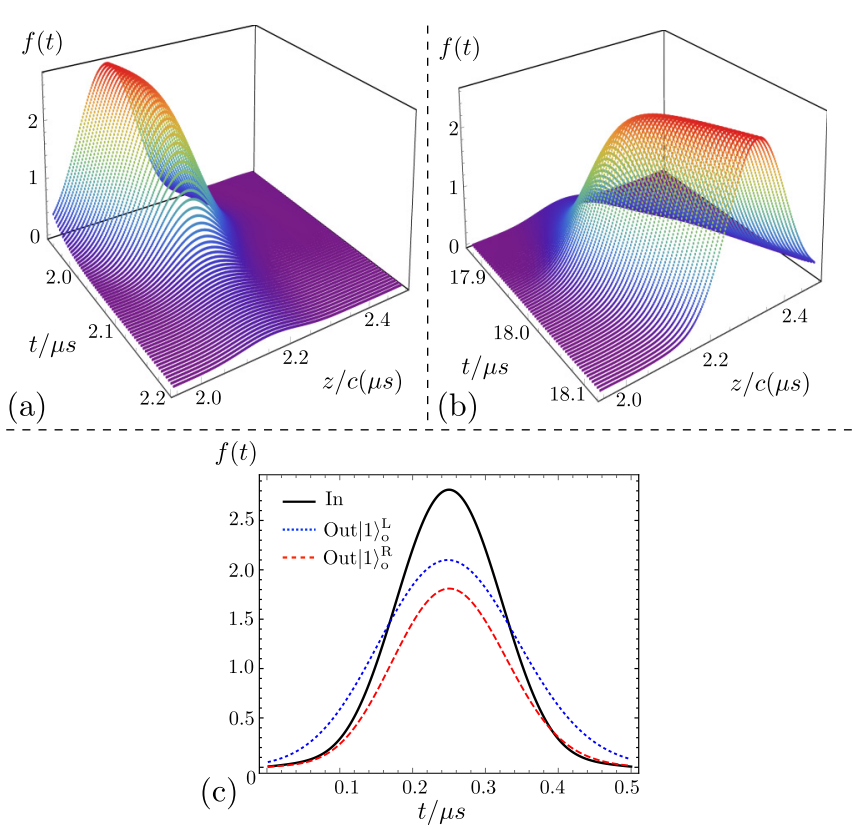

FIG. 3. Optical qubit wave-form evolution. Upper panel describes (a) the writing of optical qubit into the atom ensemble and (b) the retrieval process after the $15-\mu$ s storage time. Lower panel (c) shows the un-normalized retrieved qubit wave form $\left(|1\rangle_{o}^{L}\right.$ as blue dotted and $|1\rangle_{o}^{R}$ as red dashed), compared with the input pulse (black solid). These wave forms need to be normalized for the postselection scheme.

empty when the optical qubit is $|1\rangle_{R}$, we have $g_{m}=0$ and the input-output relation reduces to the form of a simple cavity, with the phase shift $\approx \pi$. It is important to note that the linear approximation $\left\langle\sigma_{z}\right\rangle \approx-1 / 2$ is important because a Gaussian qubit wave form can only preserve its mode shape under a linear cQED dynamics. This approximation is valid in the strong-coupling region with large cooperativity $g_{m}^{2} / \kappa \gamma_{s}$, which creates a stronger Purcell effect, and the intracavity quanta is small, which means a weak excitation of the atomic transition. This approximation is thoroughly discussed in Appendix D, and the deviation from the linear approximation would degrade the fidelity of the quantum gate, which will be simulated in Sec. VI.

\section{LOSS AND MODE SHAPE DISTORTION}

The field operator evolution formulas allow the analysis on the qubit wave form. A single-photon quantum state can be described as

$$
|\Psi(t)\rangle_{o}=\int d z f(z, t) \hat{\epsilon}^{\dagger}(z)|\mathrm{vac}\rangle=\int \mathrm{dkf}(\mathrm{k}, \mathrm{t}) \hat{\epsilon}^{\dagger}(\mathrm{k})|\mathrm{vac}\rangle,
$$

where $f(z, t)$ is the normalized state wave form, with its Fourier conjugate $f(k, t)$, which can be determined by Eq. (B15). The wave-form evolution of the optical qubit during the writing and retrieving process is simulated using the parameters listed in the previous sections with the results shown in Fig. 3.
In our protocol the two EIT channels can have different control and single-photon Rabi frequencies. Therefore, the wave form of optical qubits with different polarizations could have different phase delays and distortions, which are carefully discussed in the Appendix B. The result shows that for $|1\rangle_{o}^{R}$ qubit, the main distortion is contributed by the spin wave loss, which is an amplitude reduction of the wave form, while for $|1\rangle_{o}^{L}$ the distortion is mainly contributed by the dispersive broadening (as shown in Fig. 3). The impact of different waveform distortion mechanisms can be summarized as follows.

(1) The phase delay differences between qubits with two different polarizations can be compensated by inserting a phase plate or adjusting the two control field strengths so that the amplitude of control Rabi frequencies satisfies $\Omega_{0}^{L} / \Omega_{0}^{R}=$ $g_{L} / g_{R}$. (2) The $A$ term on the right-hand side of Eq. (4) contributes a global amplitude reduction $\sim e^{-\eta \gamma_{r_{1} / g^{\prime} g} T}$ over the outgoing wave form. For the $|1\rangle_{o}^{L / R}$ states stored in the spin waves $\left|g^{\prime}\right\rangle-|g\rangle$ and $|g\rangle-\left|r_{1}\right\rangle$, we have $\gamma_{g^{\prime} g} \ll \gamma_{g r_{1}}$. This means that the decreasing gate efficiency affects the $|1\rangle_{o}^{R}$ state more than the $|1\rangle_{o}^{L}$ state, which is shown in Fig. 3. (3) The $C / D$ terms of Eq. (4) lead to a $k$-dependent loss or dispersion. Using our sampling parameters, for an input Gaussian wave form with width $\sigma$ in the spatial domain, the $C$ term would create wave form broadened by a factor of 1.38 and 1.09 for $|1\rangle_{L}$ and $|1\rangle_{R}$, respectively. On the contrary, the $10^{-4}$ relative distortion due to the $D$-term effect is negligible.

The effect of the spin wave loss to the optical qubit state comes from the entanglement between the qubit state and the environmental noise state, which can be phenomenologically written as

$$
|\Psi\rangle_{\text {out }}^{i}=C_{1}^{o i}|1\rangle_{\text {qout }}^{i} \otimes|0\rangle_{n}^{i}+C_{2}^{o i}|0\rangle_{\text {qout }}^{i} \otimes|1\rangle_{n}^{i},
$$

where the $i=L / R$ represents the left/right polarizations, and the $C_{1,2}^{o i}$ are given by integrating Eq. (6),

$$
\left|C_{1}^{o i}\right|^{2}=\int d k\left|f_{\text {in }}(k, T) C_{1}^{o i}(k)\right|^{2},
$$

and satisfy $\left|C_{1}^{o i}\right|^{2}+\left|C_{2}^{o i}\right|^{2}=1$, which is the normalization condition. Such a qubit-noise entanglement leads to decoherence. Since the photonic qubit is a single-photon state, therefore a postselection scheme can be implemented. Generally speaking, qubits with different polarizations could suffer different losses, that is, $C_{1}^{o L} \neq C_{1}^{o R}$. For an input state $|\Psi\rangle=$ $\left(|1\rangle_{\text {in }}^{L}+|1\rangle_{\text {in }}^{R}\right) / \sqrt{2}$, the output state after the postselection with $C_{1}^{o L} \neq C_{1}^{o R}$ is apparently deviated from the ideal case. Therefore, to obtain a high gate fidelity after the postselection one can balance the loss of the two different polarizations [2] (using two ladder EIT systems, for example), at the cost of decreasing the postselection probability [70].

For a MW cat-state qubit $\left|\alpha_{\text {in/out }}\right\rangle \pm\left|-\alpha_{\text {in/out }}\right\rangle$ with

$\left|\alpha_{\text {in } / \text { out }}\right\rangle=e^{-\alpha_{0 / 1}^{2} / 2} \exp \left[\alpha_{0 / 1} \int d x f_{\text {in/out }}(x) \hat{b}_{\text {in/out }}^{\dagger}(x)\right]|0\rangle$,

where $\alpha_{0 / 1}$ is the input/output coherent amplitude and $f_{\text {in/out }}(x, t)$ is the corresponding mode shape. The MW cavity response, loss, and the lifetime of the Rydberg states affect the qubit mode shape, of which the latter two factors also introduce qubit decoherence. Using Eqs. (8) and (9), the output 

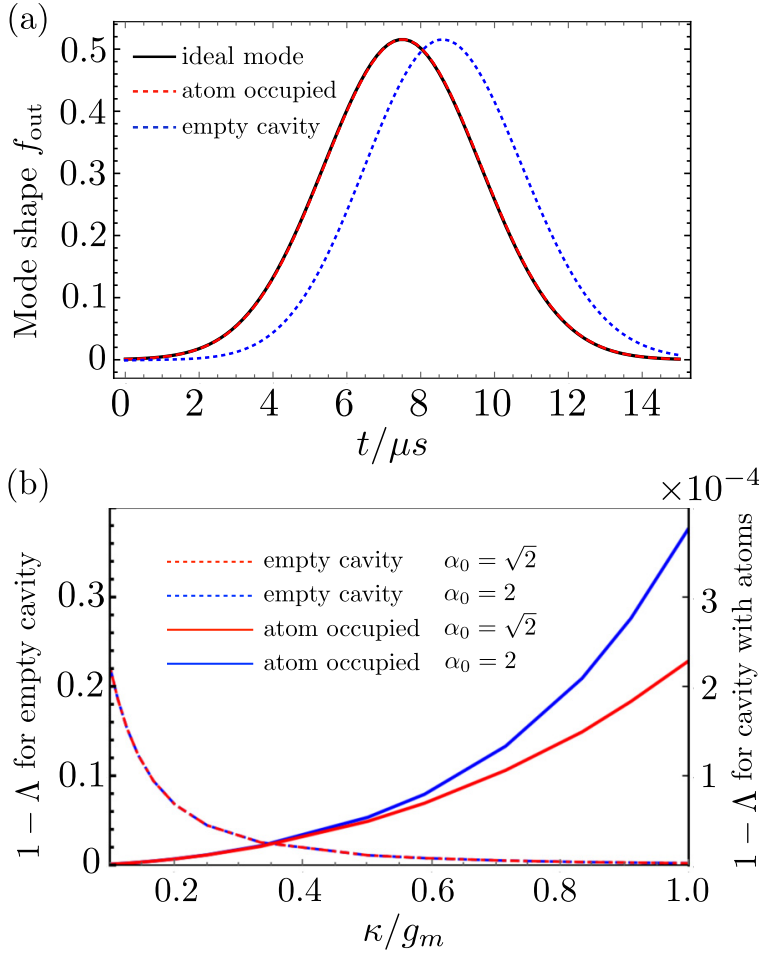

FIG. 4. (a) Mode shape of output MW qubits when $\kappa / g_{m}=0.2$ and the cavity is lossless. (b) The effect of coupling rate $\kappa$ to the phase variation and mode shape error between input and output of a lossless cavity. The values of $g_{m}, \gamma_{s}$ are set to be $2 \pi \times 2.72 \mathrm{MHz}$, $2 \pi \times 4.78 \mathrm{kHz}$.

MW qubit-noise joint state can be written as

$$
|\Psi\rangle_{\text {out }}^{\mathrm{MW}}=\left|\Psi_{+\alpha}\right\rangle_{\text {out }}^{\mathrm{MW}} \otimes\left|E_{\alpha}\right\rangle+\left|\Psi_{-\alpha}\right\rangle_{\text {out }}^{\mathrm{MW}} \otimes\left|E_{-\alpha}\right\rangle,
$$

where the qubit state $\left|\Psi_{ \pm \alpha}\right\rangle_{\text {out }}^{\mathrm{MW}}$ and the environmental state $\left|E_{ \pm \alpha}\right\rangle$ are given as

$$
\begin{aligned}
\left|\Psi_{ \pm \alpha}\right\rangle_{\text {out }}^{\mathrm{MW}} & =\exp \left[ \pm \alpha \int d \omega f_{\text {in }}(\omega, t)\left[C_{1}(\omega) \hat{b}_{\text {in }}^{\dagger}(\omega)\right]|0\rangle_{q},\right. \\
\left|E_{ \pm \alpha}\right\rangle & =\exp \left[ \pm \alpha \int d \omega f_{\text {in }}(\omega, t) C_{2}(\omega) n_{\text {in }}^{\dagger}(\omega)\right]|0\rangle_{n},
\end{aligned}
$$

and the output mode shape in the spatial domain is

$$
f_{\text {out }}(x, t)=\int d \omega f_{\text {in }}(\omega, t) C_{1}(\omega) e^{i \omega x / c} .
$$

We define the MW mode shape distortion as $1-\Lambda=$ $1-\int d x f_{\text {in }}^{*}(x) f_{\text {out }}(x)$. For an occupied Rydberg state and in the strong-coupling region, we have $\hat{b}_{\text {out }} \approx-\hat{b}_{\text {in }}$; thereby the mode shape suffers negligible distortion, i.e., $1-\Lambda \approx 0$. However, for an unoccupied Rydberg state, an empty cavity with a smaller bandwidth has a stronger response to the input qubit. This is why $1-\Lambda$ decreases with the increase of $\kappa$ in Fig. 4.

\section{FIDELITY}

Gate fidelity considering the environmental noise is obtained by $\mathscr{F}=\left\langle\Psi_{\text {ideal }}\left|\operatorname{Tr}_{E}\left[\hat{\rho}_{\text {out }}\right]\right| \Psi_{\text {ideal }}\right\rangle$ [71], in which we

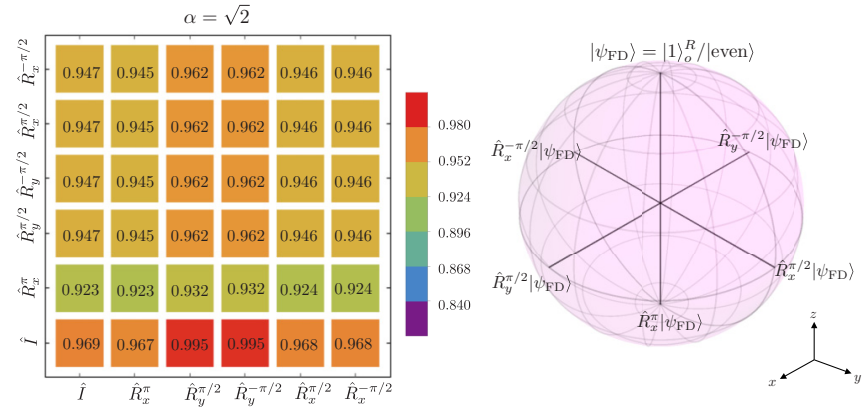

FIG. 5. The fidelity of the hybrid quantum gate with input product states when $\kappa_{s} / \kappa=10^{-3}$. The horizontal and vertical axis represent optical and MW qubit states, respectively. The color represents the fidelity values. For illustrative purposes, we define a fiducial state $|\psi\rangle_{\mathrm{FD}}=|1\rangle_{o}^{R} / \mid$ even $\rangle$, and other states are represented rotations $\hat{R}_{j}^{\theta}$ on the Bloch sphere, where $j, \theta$ are the axis index and the rotation angle.

trace out the noise channel in the output density matrix. Figure 5 shows the fidelities (considering postselection scheme for optical qubits) for those processes with separable input MW and optical states. To demonstrate the quantum nature of our CZ gate, we represent the input product states by rotations in the MW/optical Bloch sphere $|\psi\rangle_{\text {in }}=|\psi\rangle_{q}^{o} \otimes|\psi\rangle_{q}^{\mathrm{MW}}=$ $\hat{R}_{i}^{\theta}|1\rangle_{o}^{R} \otimes \hat{R}_{j}^{\theta^{\prime}} \mid$ even $\rangle$ (see Fig. 5) and the gate operations create entanglement between optical and the MW state. In computing the fidelity, we choose the MW cavity loss to be $\kappa_{s} / \kappa=$ 0.001 [72] and $\alpha=\sqrt{2}$, and we leave the mathematical details of fidelity calculation to Appendix E.

The fidelity after the postselection is degraded by the following factors: (1) the normalized wave-form distortion of output optical qubits; (2) the decoherence introduced by MW cavity loss and the MW mode shape distortion introduced by the cavity dispersive response (see Fig. 4); and (3) the MW mode shape distortion due to the cQED nonlinearity. The effect of the latter two factors is plotted in Fig. 6, where the

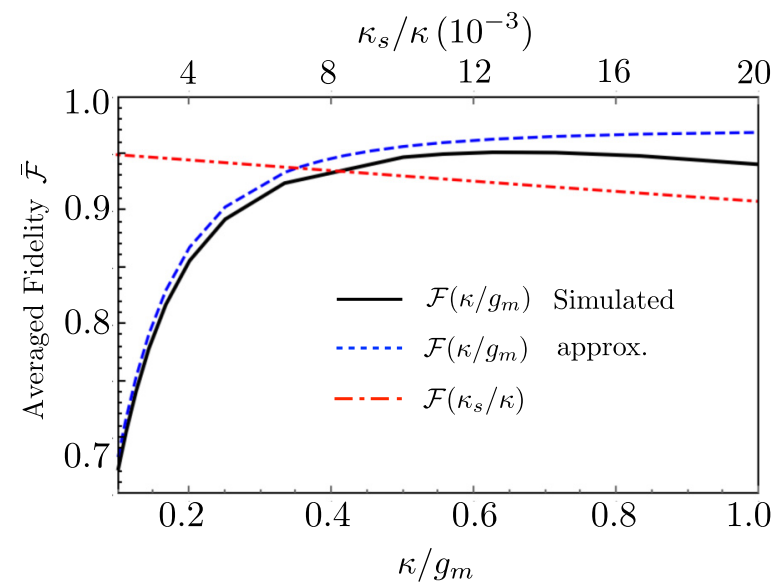

FIG. 6. The averaged fidelity of the hybrid quantum $\mathrm{CZ}$ gate shown as a function of cavity loss $\kappa_{s}$ and cavity bandwidth $\kappa$, when $g_{m}=2 \pi \times 2.72 \mathrm{MHz}, \kappa=2 \pi \times 2.0 \mathrm{MHz}$, and $\gamma_{s}=2 \pi \times$ $4.78 \mathrm{kHz}$. The solid line and the dashed line are the result obtained by the full cQED simulation and linear approximation $\left\langle\hat{\sigma}_{z}\right\rangle=-1 / 2$. 
average fidelity $\overline{\mathscr{F}}$ is obtained by averaging over those input states shown in Fig. 5. Moreover, in the Appendixes we show the fidelity for Schrödinger cat state with different coherent amplitudes $\alpha$.

\section{CONCLUSION AND OUTLOOK}

In summary, we have proposed and analyzed a protocol for the realization of an optical-microwave hybrid quantum $\mathrm{CZ}$ gate utilizing a Rydberg-based atomic medium. The gate performance under various noises is studied, and we conclude that high gate fidelity in principle can be achieved with current state-of-the-art parameters. From our analysis on the parameter settings (for details, see the Appendixes), the main experimental challenges for this scheme are (i) the low decoherence rate of Rydberg states requires either spatial confinement of Rydberg atoms or employ ultracold atoms, and (ii) a high-Q microwave resonator compatible with Rydberg atoms to reach the strong cavity-atom coupling.

Our result indicates that with the development of quantum science and technology, such a hybrid quantum gate could find important applications in future distributed quantum networks. For example, as discussed in the Appendixes, one can use a photon as an ancilla qubit and combine our hybrid gate for the realization of a remote quantum logic gate between MW qubits in distant quantum modules. Such a remote quantum connectivity is indispensable for distributed quantum computing with superconducting circuits. Beyond these specific applications, our work also adds a component to the Rydberg quantum optics toolbox by demonstrating the possibility to use Rydberg atoms for the coherent quantum control between photons at completely different frequency bands.

\section{ACKNOWLEDGMENTS}

L.L. and Y.M. are supported by university start-up fundings provided by Huazhong University of Science and Technology. The authors thank Daiqin Su, Saijun Wu, Shengjun Yang, Kuan Zhang, Shuai Shi, Zhiyuan Wei, Yafen Cai, Zebing Zhou, and Yanbei Chen for helpful discussions. The authors thank Yuelong Liu for administrative support. L.L. was also supported by the National Natural Science Foundation of China (Grant No. 12004127).

\section{APPENDIX A: HAMILTONIAN FOR 1D FREE-PROPAGATING EM FIELDS}

The Hamiltonian describes a one-dimensional, freely propagating quantum electromagnetic (EM) field derived as follows. In the frequency domain, the general form of the free EM Hamiltonian is

$$
H_{f}=\hbar c \int_{0}^{\infty} \mathrm{d} k|k| \hat{a}_{k}^{\dagger} \hat{a}_{k} .
$$

For a unidirectional field pulse with center spatial frequency $k_{0}$, it can be written as

$$
H_{f}=\hbar c \int_{-k_{0}}^{\infty} \mathrm{d} k\left(k_{0}+k\right) \hat{a}_{k_{0}+k}^{\dagger} \hat{a}_{k_{0}+k} .
$$

Since we are interested in the case $k \ll k_{0}$, we can approximate the above formula as

$$
H_{f}=\hbar c \int_{-\infty}^{+\infty} \mathrm{d} k\left(k_{0}+k\right) \hat{a}_{k_{0}+k}^{\dagger} \hat{a}_{k_{0}+k} .
$$

Now we define the Fourier transform of the field operator in $z$ space:

$$
\hat{a}_{z}=\int_{-\infty}^{+\infty} \hat{a}_{k_{0}+k} e^{-i k z} \mathrm{~d} k, \quad \hat{a}_{k_{0}+k}=\int_{-\infty}^{+\infty} \hat{a}_{z} e^{i k z} \mathrm{~d} z .
$$

Substituting them into Eq. (A3) and choosing rotating frame $e^{-i k_{0} z}$ we obtain

$$
H_{f}=\frac{i \hbar c}{2} \int_{-\infty}^{+\infty} \mathrm{d} z\left[\frac{\partial \hat{a}_{z}^{\dagger}}{\partial z} \hat{a}_{z}-\frac{\partial \hat{a}_{z}}{\partial z} \hat{a}_{z}^{\dagger}\right] .
$$

If the quantization volume has length $L$, the quantum optical field operator can be redefined as $\hat{\varepsilon}=\sqrt{L} \hat{a}_{z}$, which leads to

$$
H_{f}=\frac{i \hbar c}{2 L} \int_{-\infty}^{+\infty} \mathrm{d} z\left[\frac{\partial \hat{\varepsilon}^{\dagger}}{\partial z} \hat{\varepsilon}-\frac{\partial \hat{\varepsilon}}{\partial z} \hat{\varepsilon}^{\dagger}\right],
$$

which is the Hamiltonian we used in the main text.

\section{APPENDIX B: DERIVATION OF THE OPTICAL QUBIT EVOLUTION}

In the model discussed in the main text, the weak probe field $\hat{\epsilon}$ resonantly couples to $|b\rangle-|a\rangle$, a coherent control field with Rabi frequency $\Omega(z, t)$ couples to the excited level $|a\rangle-$ $|c\rangle$, and the Hamiltonian in the interaction picture is [73]

$$
\begin{aligned}
H= & \frac{i \hbar c}{2 L} \int_{-\infty}^{+\infty} \mathrm{d} z\left[\frac{\partial \hat{\varepsilon}^{\dagger}}{\partial z} \hat{\varepsilon}-\frac{\partial \hat{\varepsilon}}{\partial z} \hat{\varepsilon}^{\dagger}\right] \\
& -\hbar \sum_{j=1}^{N}\left[\Omega\left(z_{j}, t\right) \hat{\sigma}_{a c}^{j}+g \hat{\varepsilon} \hat{\sigma}_{b c}^{j}+\text { H.c. }\right],
\end{aligned}
$$

where $g$ is the atom-field coupling constant, and $\hat{\sigma}_{\mu v}=|\mu\rangle\langle v|$ is the transition operator of the atom from $|v\rangle$ to $|\mu\rangle$.

To write it in a continuous form, we can introduce the following operator:

$$
\hat{\sigma}_{\mu v}(z, t)=\frac{1}{n(z)} \sum_{j=1}^{n(z)} \hat{\sigma}_{\mu v}^{j}(z, t),
$$

where $n(z) \gg 1$ is the sum number of atoms at position $z$, and $\sum_{j=1}^{N}$ can be replaced with $\int \mathrm{d} z n(z)$ in the continuum limit. Using these continuous atom-field operators, the Hamiltonian can be written as

$$
\begin{aligned}
\hat{H}= & \frac{i \hbar c}{2 L} \int_{-\infty}^{+\infty} \mathrm{d} z\left[\frac{\partial \hat{\varepsilon}^{\dagger}}{\partial z} \hat{\varepsilon}-\frac{\partial \hat{\varepsilon}}{\partial z} \hat{\varepsilon}^{\dagger}\right]-\int_{0}^{L} \mathrm{~d} z n(z) \\
& \cdot\left[\hbar g \hat{\sigma}_{a b}(z, t) \hat{\varepsilon}(z, t)+\hbar \Omega(z, t) \hat{\sigma}_{a c}(z, t)+\text { H.c. }\right],
\end{aligned}
$$

where we assume that the atom's ensemble has the same length as $L, N$ is the total number of atoms, and $n(z)$ is the linear atom number density in propagation direction. The communication relations of the atomic and field operators are

$$
\begin{aligned}
& {\left[\hat{\sigma}_{\mu v}(z, t), \hat{\sigma}_{\alpha \beta}\left(z^{\prime}, t\right)\right]} \\
& =\frac{1}{n(z)}\left[\hat{\sigma}_{\mu \beta}(z, t) \delta_{v \alpha}-\hat{\sigma}_{v \alpha}(z, t) \delta_{\mu \beta}\right] \delta\left(z-z^{\prime}\right), \\
& \quad \times\left[\hat{\epsilon}(z, t), \hat{\epsilon}^{\dagger}\left(z^{\prime}, t\right)\right]=L \delta\left(z-z^{\prime}\right) .
\end{aligned}
$$


The corresponding Heisenberg-Langevin equations are

$$
\begin{aligned}
& \dot{\hat{\sigma}}_{b a}=-\gamma_{b a} \hat{\sigma}_{b a}+i g \hat{\varepsilon}\left(\hat{\sigma}_{b b}-\hat{\sigma}_{a a}\right)+i \Omega \hat{\sigma}_{b c}+\hat{F}_{b a}, \\
& \dot{\hat{\sigma}}_{b c}=-\gamma_{b c} \hat{\sigma}_{b c}+i \Omega^{*} \hat{\sigma}_{b a}-i g \hat{\varepsilon} \hat{\sigma}_{a c}+\hat{F}_{a c},
\end{aligned}
$$

where $\gamma_{\mu v}$ is the atom decay rate from level $v$ to $\mu$, and $\hat{F}_{\mu v}$ is corresponding Langevin noise operators.

The optical qubit propagation equation is as [where we assume the atomic medium is homogeneous, i.e., $n(z)=$ const. and $n(z) L=N]$

$$
\left(\frac{\partial}{\partial t}+c \frac{\partial}{\partial z}\right) \hat{\epsilon}(z, t)=i g N \hat{\sigma}_{b a}(z, t) .
$$

For the proposal discussed in the main text, the initial state of the first-order perturbation of the equations of motion is

$$
\begin{aligned}
& \dot{\hat{\sigma}}_{b a}=-\gamma_{b a} \hat{\sigma}_{b a}+i g \hat{\epsilon}+i \Omega \hat{\sigma}_{b c}+\hat{F}_{b a}, \\
& \dot{\hat{\sigma}}_{b c}=-\gamma_{b c} \hat{\sigma}_{b c}+i \Omega^{*} \hat{\sigma}_{b a}+\hat{F}_{a c} .
\end{aligned}
$$

Since the Raman process effectively only involve the two hyperfine structure levels, we eliminate the excited level and obtain

$$
\begin{aligned}
\left(\frac{\partial}{\partial t}+c \frac{\partial}{\partial z}\right) \tilde{\varepsilon}(z, t)= & \frac{g N}{\Omega^{*}}\left(\frac{\partial}{\partial t}+\gamma_{b c}\right) \tilde{\sigma}_{b c}-\frac{g N}{\Omega^{*}} \hat{F}_{a c}, \\
\tilde{\sigma}_{b c}= & -\frac{g \tilde{\varepsilon}}{\Omega}-\frac{i}{\Omega}\left(\frac{\partial}{\partial t}+\gamma_{b a}\right) \\
& {\left[-\frac{i}{\Omega^{*}}\left(\frac{\partial}{\partial t}+\gamma_{b c}\right) \tilde{\sigma}_{b c}\right] } \\
& +\frac{1}{\Omega}\left(\frac{\partial}{\partial t}+\gamma_{b a}\right) \frac{F_{b c}}{\Omega^{*}}+\frac{i F_{b a}}{\Omega} .
\end{aligned}
$$

Now we perform the adiabatic approximation to these equations. Basically, the coherent control field is switch on and off slowly enough to avoid the transition from a dark to bright state. This means the terms related to time evolution of Eq. (B8) can be ignored, and the adiabatic condition can be expressed as

$$
\left|\frac{1}{\Omega} \frac{\partial \Omega}{\partial t}\right| \ll \frac{|\Omega|^{2}}{\gamma_{b a}},\left|\frac{1}{\Omega} \frac{\partial \Omega}{\partial t}\right| \ll|\Omega|,\left|\frac{1}{\Omega} \frac{\partial^{2} \Omega}{\partial t^{2}}\right| \ll|\Omega|^{2},
$$

which leads to

$$
\left(\frac{\partial}{\partial t}+c \frac{\partial}{\partial z}\right) \hat{\varepsilon}(z, t)=\frac{g N}{\Omega^{*}} \frac{\partial \hat{\sigma}_{b c}}{\partial t}, \quad \hat{\sigma}_{b c}=-\frac{g \hat{\varepsilon}}{\Omega} .
$$

Further eliminating the $\hat{\sigma}_{b c}$ gives

$$
\left(\frac{\partial}{\partial t}+c \frac{\partial}{\partial z}\right) \hat{\varepsilon}(z, t)=\frac{g^{2} N}{|\Omega|^{2} \Omega} \frac{\partial \Omega}{\partial t} \hat{\varepsilon}-\frac{g^{2} N}{|\Omega|^{2}} \frac{\partial \hat{\varepsilon}}{\partial t},
$$

which can be written as

$$
\left(\frac{\partial}{\partial t}+v \frac{\partial}{\partial z}\right) \tilde{\varepsilon}(z, t)=\beta \tilde{\varepsilon}(z, t) .
$$

Here we define refractive index $n_{g}=1 /(1-\eta)$ with $\eta=$ $g^{2} N /\left(g^{2} N+|\Omega|^{2}\right)$, where the group velocity $v$ and the decay rate $\beta$ are

$$
v=c / n_{g}, \quad \beta=\frac{\eta}{\Omega} \frac{\partial \Omega}{\partial t} .
$$

These equations describe an ideal EIT storage. When we reduce the control light, the speed of $\tilde{\epsilon}$ is slowed down, which is the EIT slow-light effect. The writing and retrieving processes correspond to $\beta<0$ and $\beta>0$, where the control field is switched off and on adiabatically, respectively.

Next we analyze the imperfections of the EIT storage, e.g., the nonadiabaticity and finite $\gamma_{b c}, \gamma_{b a}$. We approximate $\tilde{\sigma}_{b c}$ as $g \hat{\varepsilon} / \Omega$, which leads to

$$
\begin{aligned}
\tilde{\sigma}_{b c}= & -\frac{g \hat{\varepsilon}}{\Omega}-\frac{i}{\Omega}\left(\frac{\partial}{\partial t}+\gamma_{b a}\right)\left[-\frac{i}{\Omega^{*}}\left(\frac{\partial}{\partial t}+\gamma_{b c}\right) \frac{g \hat{\varepsilon}}{\Omega}\right] \\
& +\frac{1}{\Omega}\left(\frac{\partial}{\partial t}+\gamma_{b a}\right) \frac{\hat{F}_{b c}}{\Omega^{*}}+\frac{i \hat{F}_{b a}}{\Omega} .
\end{aligned}
$$

Replacing $\hat{\sigma}_{b c}$ in Eq. (B8) by the above formula and keep the leading terms, we obtain Eqs. (4-5) in the main text. However, after the optical field thoroughly passed the atom ensemble, the noise fields which broaden the atom state should be added to $\hat{\epsilon}$ for compensating the bosonic commutation relation. The optical field operator can be written, after passing the atom ensemble (after time $T$ ),

$$
\begin{aligned}
\hat{\varepsilon}(k, T)= & \hat{\varepsilon}(k, 0) \exp \left\{i \int_{0}^{T} \mathrm{~d} t\left[k v-k^{3} c^{3} D(1-\eta)^{3}\right]\right\} \\
& \exp \left\{\int_{0}^{T} \mathrm{~d} t\left[A-k^{2} c^{2}(1-\eta)^{2} C\right]\right\}+\text { noise terms, }
\end{aligned}
$$

with

$A=\eta\left[\frac{1}{\Omega} \frac{\partial \Omega}{\partial t}-\gamma_{r_{1} / g^{\prime} g}\right], \quad D=\frac{\eta}{|\Omega|^{2}}$,

$C=\frac{\eta}{|\Omega|^{2}}\left[\left(2 \gamma_{r_{1} / g^{\prime} g}+\gamma_{e g}\right)-2\left(\frac{1}{\Omega^{*}} \frac{\partial \Omega^{*}}{\partial t}+\frac{2}{\Omega} \frac{\partial \Omega}{\partial t}\right)\right]$.

Here the first exponential factor is a phase shift [denoted as $\left.\Phi_{T}(k)\right]$, in which the term $\sim k v$ describes the simple propagation of the qubit pulse in the atomic medium with velocity $v=c / n_{g}$, while the $D$ term is the pulse shape distortion due to dispersion. The second exponential factor is an attenuation factor [defined as $\chi(k)$ ].

As a bosonic operator, $\hat{\epsilon}$ should satisfy the bosonic commutation relation, thereby other degrees of freedom should be added onto the above formula to conserve the phase space [74]. Therefore one can treat the noise term using the method that phenomenologically writes down the noise term with the coefficient determined by the bosonic commutation relation, which was developed by Caves [69] for general quantum amplifiers. For example, for the storage process the atom operators should be added since the optical field and spin wave superposed to be the polaritons. However, for the output optical field from the atom ensemble, we should add to $\hat{\epsilon}$ those noise fields $\hat{n}$ which are associated with various loss channels and we have

$$
\hat{\epsilon}(k, T)=\chi(k) e^{i \Phi_{T}(k)} \hat{\epsilon}(k, 0)+\sqrt{1-\chi(k)^{2}} \hat{n}_{k},
$$

where $\hat{n}$ describes the noise corresponding to all possible losses. In the following, we will give some discussions on the physical content of this formula.

Relative phase. In our protocol the two EIT channels could have different control Rabi frequencies denoted as $\Omega_{R / L}$, and 
they also have different single-photon Rabi frequencies $g_{R / L}$. Therefore the optical qubits with different polarizations could have different phases and distortions, which leads to different wave-form distortions. Now we discuss these effects as follows.

The relative propagation phase $\sim k\left(v_{L}-v_{R}\right)$ between two polarizations is

$$
\Delta \Phi=k c \int_{0}^{T}\left(\frac{\left|\Omega_{L}(t)\right|^{2}}{\left|\Omega_{L}(t)\right|^{2}+g_{L}^{2} N}-\frac{\left|\Omega_{R}(t)\right|^{2}}{\left|\Omega_{R}(t)\right|^{2}+g_{R}^{2} N}\right) d t,
$$

in which the $\Omega_{R / L}$ is

$$
\begin{gathered}
\Omega_{R / L}(t)=\Omega_{0}^{R / L} f_{\Omega}(t) \\
f_{\Omega}(t)=\frac{1}{2}\left(2+\tanh \left[20(t-T]-\tanh \left[20\left(t-t_{0}\right)\right]\right) .\right.
\end{gathered}
$$

To eliminate the phase difference, there are two methods: (1) we can simply set

$$
\Omega_{0}^{L} / \Omega_{0}^{R}=g_{L} / g_{R}
$$

in Eq. (B18) so that the relative phase of the two polarizations would be canceled; (2) if the above condition does not satisfy, we can introduce a compensation phase in the input/output path of the optical qubit by applying a phase plate. For example, if we choose $\Omega_{0}^{L}=\Omega_{0}^{R}=2 \pi \times 30 \mathrm{MHz}$, our calculations show the compensation phase is $0.936 \pi \mathrm{rad}$.

Wave-form distortions. In Fig. 3 of the main text, we have shown the wave-form distortions of the optical quit pulses due to the dispersive and loss terms in Eq. (B15), in comparison with the input wave form. For revealing the distortion details, let us look at Eq. (B16).

Firstly, it is easy to see that the $A$ term in Eq. (B16) contributes a global amplitude reduction $\sim e^{-\eta \gamma_{r_{1} / g^{\prime} g} T}$ over the wave form of the outgoing field. For the $|1\rangle_{o}^{L}$ and $|1\rangle_{o}^{R}$ states that are stored in the spin waves $\left|g^{\prime}\right\rangle-|g\rangle$ and $|g\rangle-\left|r_{1}\right\rangle$, we have $\gamma_{g^{\prime} g} \ll \gamma_{g r_{1}}$. This means that the amplitude of $|1\rangle_{o}^{R}$ is globally smaller than that of $|1\rangle_{o}^{L}$ over the pulse duration. This reduction will decrease the gate efficiency, which can be characterized by $\zeta=\int_{-\infty}^{\infty} d x\left|f_{\text {out }}(x)\right|^{2}$. Using our sampling parameters, we have $\zeta=0.997$ for $|1\rangle_{o}^{L}$ and $\zeta=0.492$ for $|1\rangle_{o}^{R}$, respectively.

Secondly, the $C / D$ terms in Eq. (B16) contribute a $k$ dependent loss/dispersion which will distort the wave-form shape. For the $|1\rangle_{o}^{R / L}$ qubit, the $k$-dependent loss is mainly contributed by $\gamma_{e g}$ in the $C$ term since $\gamma_{r_{1} / g^{\prime} g} \ll \gamma_{e g}$. For example, for an input Gaussian profile with wave function $f_{\text {in }}(k)=e^{-k^{2} / 4 \sigma_{k}^{2}} / \sqrt{2 \pi}$, the $C$ term could lead to a diffusion as

$$
\begin{aligned}
f_{\text {in }}(k) & \rightarrow \frac{1}{\sqrt{2 \pi} \sigma_{k}} e^{-k^{2} / 4 \sigma_{k}^{2}} \exp \left[-k^{2} \int_{0}^{T} d t c^{2}\left(1-\eta^{2}\right) C\right] \\
& =\frac{1}{\sqrt{2 \pi} \sigma_{k}} \exp \left[-\frac{k^{2}}{4 \tilde{\sigma}_{k}^{2}}\right],
\end{aligned}
$$

where

$$
\frac{1}{\tilde{\sigma}_{k}^{2}}=\frac{1}{\sigma_{k}^{2}}+4 \int_{0}^{T} d t c^{2}\left(1-\eta^{2}\right) C
$$

Apparently, the squared variance decreases/increases in the $k$ spatial domain. Using the example parameters in our simulation, the ratio $\tilde{\sigma}_{k} / \sigma_{k}$ for $|1\rangle_{o}^{L}$ and $|1\rangle_{o}^{R}$ is 1.38 and 1.09, which is consistent with the result shown in Fig. 3 of the main text. The gate efficiency reduced by the $C$ term is $\zeta=0.745$ for $|1\rangle_{o}^{L}$ and $\zeta=0.928$ for $|1\rangle_{o}^{R}$, respectively. Combining the gate efficiency reduced by the $C$ and $A$ terms leads to the gate efficiency provided in the truth table of the main text.

The influence of the $D$ term to the wave form is relatively small; a simple estimation using our sampling parameters can easily show that we can approximate the influence of the $D$ term by Taylor expansion as

$f_{\text {in }}(k) \rightarrow \frac{1}{\sqrt{2 \pi} \sigma_{k}} e^{-k^{2} / 4 \sigma_{k}^{2}}\left[1-i k^{3} c^{3} \int_{0}^{T} d t D(1-\eta)^{3}\right]$.

Fourier transformation to the spatial domain leads to the $D$ correction term to the original wave form as

$$
8 \sqrt{\pi} i \sigma_{k}^{4}\left[2\left(\sigma_{k} x\right)^{3}-3 \sigma_{k} x\right] e^{-\sigma_{k}^{2} x^{2}} .
$$

Using the parameters in our simulation and setting $x \sim 1 / \sigma_{k}$, the orders of magnitude of the ratio between the correction term Eq. (B25) and the input wave form is $4.67 \times 10^{-4}$ and $2.70 \times 10^{-4}$ for $|1\rangle_{o}^{L}$ and $|1\rangle_{o}^{R}$, respectively, from which we can understand that the $D$-term corrections are very small compare to the distortion contributed by the $C$ term.

In a short summary, we can conclude that for $|1\rangle_{o}^{R}$, the distortion is mainly contributed by the $A$ term, which is a global amplitude reduction of the wave form, e.g., for $|1\rangle_{o}^{L}$ the distortion is mainly contributed by the $C$ term.

Phenomenological description of the qubit states. For a single-photon state, the output optical quantum state now is ( $\hat{U}$ is the evolution operator)

$$
\begin{aligned}
|\Psi\rangle_{\text {out }}= & \hat{U} \int d x f_{\text {in }}(x) \hat{\epsilon}_{x}^{\dagger}|\mathrm{vac}\rangle=\hat{U} \int d k f_{\text {in }}(k) \hat{\epsilon}_{k}^{\dagger}|\mathrm{vac}\rangle \\
= & \int d k f_{\text {in }}(k, T)\left[\chi^{*}(k) e^{-i \Phi_{T}(k)} \hat{\epsilon}_{k}^{\dagger}(0)\right. \\
& \left.+\sqrt{1-|\chi(k)|^{2}} \hat{n}_{k}^{\dagger}\right]|\mathrm{vac}\rangle,
\end{aligned}
$$

where we have used the fact that $\hat{U}|\mathrm{vac}\rangle=|\mathrm{vac}\rangle$, and the $|\mathrm{vac}\rangle$ should be the joint Hilbert space of optical qubit and the noise field. In a more phenomenological way, it can be written as (despite the unimportant common phase for $R / L$ polarization, respectively):

$$
\begin{aligned}
& |\Psi\rangle_{\text {out }}^{L}=C_{1}^{o L}\left|1_{\text {outL }}\right\rangle_{q} \otimes|0\rangle_{n}^{L}+C_{2}^{o L}|0\rangle \otimes|1\rangle_{n}^{L}, \\
& |\Psi\rangle_{\text {out }}^{R}=C_{1}^{o R}\left|1_{\text {outR }}\right\rangle_{q} \otimes|0\rangle_{n}^{R}+C_{2}^{o R}|0\rangle \otimes|1\rangle_{n}^{R},
\end{aligned}
$$

with

$$
C_{1}^{o L / R}=\sqrt{\int d k\left|f_{\text {in }}(k, T)\right|^{2}\left|\chi^{L / R}(k)\right|^{2}},
$$

and $\left|C_{1}^{o L}\right|^{2}+\left|C_{2}^{o R}\right|^{2}=1$, which is the normalization condition.

Since the optical qubit here is a single-photon state, we can use the postselection detection scheme for many applications. In the postselection scheme, for the superposition input state $|\Psi\rangle=a|\Psi\rangle_{\text {in }}^{L}+b|\Psi\rangle_{\text {in }}^{R}$, we have postselected the output qubit 
state as

$$
|\Psi\rangle_{\text {out }}=a C_{1}^{o L}\left|1_{\text {outL }}\right\rangle_{q}+b C_{1}^{o R}\left|1_{\text {outR }}\right\rangle_{q} .
$$

If we have balanced the EIT storage process for the optical qubits with left and right polarizations, that is, $C_{1}^{o L}=C_{1}^{o R}$, we then have

$$
|\Psi\rangle_{\text {out }}=C_{1}^{o R}\left(a\left|1_{\text {outL }}\right\rangle_{q}+b\left|1_{\text {outR }}\right\rangle_{q}\right),
$$

which perfectly matches the ideal qubit state. Therefore, practically, we can increase the loss of certain channels to balance these two EIT storage processes at the price of sacrificing the postselection probability.

\section{APPENDIX C: CAVITY QUANTUM ELECTRODYNAMICS}

The microwave (MW) qubit is processed by a cavity QED system during the optical storage. In the above EIT process, we use single photon as optical qubits; therefore at most one atom populates on the Rydberg state $\left|r_{1}\right\rangle$. In addition, the wavelength of a MW qubit is much larger than the size of the Rydberg atom ensemble.

In this case, a MW qubit enters the cavity and reflects from the cavity. In the rotating frame, the Hamiltonian is (the mi- crowave cavity is on resonance with the transition $\left|r_{1}\right\rangle \rightarrow\left|r_{2}\right\rangle$ )

$$
H=\hbar g_{m} \hat{a}_{c} \hat{\sigma}_{r_{2} r_{1}}+\hbar \sqrt{\kappa} \hat{a}_{c} \hat{b}^{\dagger}(0)+\hbar \sqrt{\kappa} \hat{a}_{c} \hat{n}^{\dagger}+\text { H.c. }
$$

where $g_{m}$ is the coupling rate of the single atom to the cavity field, $\kappa$ represents the cavity-qubit coupling strength, while $\kappa_{s}$ represents the MW cavity loss rate.

The Heisenberg equations of motion for the cavity QED system read

$$
\begin{aligned}
& \dot{\hat{\sigma}}_{r_{1} r_{2}}=-\gamma_{s} \hat{\sigma}_{r_{1} r_{2}}+2 i g_{m} \hat{\sigma}_{z} \hat{a}_{c}+\sqrt{2 \gamma_{s}} \hat{n}_{\mathrm{en}}, \\
& \dot{\hat{a}}_{c}=-i g_{m} \hat{\sigma}_{r_{1} r_{2}}-\frac{\kappa+\kappa_{s}}{2} \hat{a}_{c}-\sqrt{\kappa} \hat{a}_{i n}-\sqrt{\kappa_{s}} \hat{n}, \\
& \hat{b}_{\text {out }}=\hat{b}_{\text {in }}+\sqrt{\kappa} \hat{a}_{c},
\end{aligned}
$$

where $\hat{n}_{\mathrm{en}}$ and $\hat{n}$ are noise induced by the decay of the Rydberg state and cavity loss, respectively. These equations can be approximately treated in the strong-coupling region and small cat-state amplitude (see Sec. D). In the Fourier domain, it has the following form:

$$
\begin{gathered}
\left(-i \omega+\gamma_{s}\right) \hat{\sigma}_{r_{1} r_{2}}(\omega)=-i g_{m} \hat{a}_{c}(\omega)+\sqrt{2 \gamma_{s}} \hat{n}_{\mathrm{en}}(\omega), \\
\left(-i \omega+\frac{\kappa+\kappa_{s}}{2}\right) \hat{a}_{c}(\omega)=-i g_{m} \hat{\sigma}_{r_{1} r_{2}}(\omega)-\sqrt{\kappa} \hat{a}_{\text {in }}(\omega)-\sqrt{\kappa_{s}} \hat{n}(\omega) .
\end{gathered}
$$

Solving these equations in the Fourier domain, we have

$$
\hat{a}_{c}(\omega)=\frac{\sqrt{\kappa} \hat{b}_{\mathrm{in}}(\omega)+\sqrt{\kappa_{s}} \hat{n}(\omega)+i g_{m} \sqrt{2 \gamma_{s}} \hat{n}_{\mathrm{en}}(\omega) /\left(-i \omega+\gamma_{s}\right)}{i \omega+g_{m}^{2} /\left(i \omega-\gamma_{s}\right)-\left(\kappa+\kappa_{s}\right) / 2},
$$

and the input-output relation is

$$
\begin{aligned}
\hat{b}_{\text {out }}(\omega)= & \frac{i \omega+g_{m}^{2} /\left(i \omega-\gamma_{s}\right)-\left(\kappa_{s}-\kappa\right) / 2}{i \omega+g_{m}^{2} /\left(i \omega-\gamma_{s}\right)-\left(\kappa_{s}+\kappa\right) / 2} \hat{b}_{\text {in }}(\omega)+\frac{\sqrt{\kappa \kappa_{s}}}{i \omega+g_{m}^{2} /\left(i \omega-\gamma_{s}\right)-\left(\kappa_{s}+\kappa\right) / 2} \hat{n}(\omega) \\
& -\frac{i g_{m} \sqrt{2 \kappa \gamma_{s}} /\left(i \omega-\gamma_{s}\right)}{i \omega+g_{m}^{2} /\left(i \omega-\gamma_{s}\right)-\left(\kappa_{s}+\kappa\right) / 2} \hat{n}_{\text {en }}(\omega) .
\end{aligned}
$$

The second and third term correspond to the noise injection from the cavity loss and the Rydberg state decay. Using Cave's approach [69], we can phenomenologically write a noise term $\propto \hat{n}_{\text {in }}$ which represents both these contributions as

$$
\hat{b}_{\text {out }}(\omega)=C_{1}(\omega) \hat{b}_{\text {in }}(\Omega)+C_{2}(\omega) \hat{n}_{\text {in }}(\omega)
$$

where $\hat{n}_{\text {in }}$ represents all possible noise contributions, and

$$
\begin{aligned}
C_{1}(\omega) & =\frac{i \omega+g_{m}^{2} /\left(i \omega-\gamma_{s}\right)-\left(\kappa_{s}-\kappa\right) / 2}{i \omega+g_{m}^{2} /\left(i \omega-\gamma_{s}\right)-\left(\kappa_{s}+\kappa\right) / 2}, \\
C_{2}(\omega) & =\frac{i \omega-\gamma_{\mathrm{eff}}}{i \omega-\gamma_{s}} \frac{\sqrt{\kappa \kappa_{s}}}{i \omega+g_{m}^{2} /\left(i \omega-\gamma_{s}\right)-\left(\kappa_{s}+\kappa\right) / 2}, \\
\left|C_{1}(\omega)\right|^{2}+\left|C_{2}(\omega)\right|^{2} & =1,
\end{aligned}
$$

with $\gamma_{\text {eff }}=\sqrt{\gamma_{s}^{2}+2 g_{m}^{2} \gamma_{s} / \kappa_{s}}$

Let us consider that the input state is a Schrödinger cat state:

$$
|\Psi\rangle_{\text {in }}^{\mathrm{MW}}=e^{-\alpha^{2} / 2}\left[\exp \left(\alpha \int d x f_{\text {in }}(x, t) \hat{b}_{\text {in }}^{\dagger}(x)\right)+(\alpha \rightarrow-\alpha)\right]|0\rangle_{q n},
$$


where $|0\rangle_{q n}=|0\rangle_{q} \otimes|0\rangle_{n}$ is the joint vacuum state of MW qubit and the noise field. The MW qubit-noise joint state evolution can be written as (we ignore the normalization factor for brevity)

$$
|\Psi\rangle_{\text {out }}=\hat{U}|\Psi\rangle_{\text {in }}^{\mathrm{MW}}=\left[\exp \left(\alpha \int d x f_{\text {in }}(x, t) \hat{b}_{\text {out }}^{\dagger}(x)\right)+(\alpha \rightarrow-\alpha)\right]|0\rangle_{\text {qn }}=\left|\Psi_{\alpha}\right\rangle_{\text {out }}^{q} \otimes\left|E_{\alpha}\right\rangle+\left|\Psi_{-\alpha}\right\rangle_{\text {out }}^{q} \otimes\left|E_{-\alpha}\right\rangle,
$$

where

$$
\left|\Psi_{ \pm \alpha}\right\rangle_{\text {out }}^{q}=\exp \left[ \pm \alpha \int d \omega f_{\text {in }}(\omega, t)\left[C_{1}(\omega) \hat{b}_{\text {in }}^{\dagger}(\omega)\right]|0\rangle_{q}\left|E_{ \pm \alpha}\right\rangle=\exp \left[ \pm \alpha \int d \omega f_{\text {in }}(\omega, t) C_{2}(\omega) n_{\text {in }}^{\dagger}(\omega)\right]|0\rangle_{n} .\right.
$$

In the spatial domain, the mode shape function $f_{\text {out }}(x, t)$ is

$$
f_{\text {out }}(x, t)=\int d \omega f_{\text {in }}(\omega, t) C_{1}(\omega) e^{i \omega x / c}
$$

The input and output pulse shapes are given in Fig. 4 of the main text. For the case when the Rydberg state is occupied, the mode function mismatch between the input and output fields are negligible. However, when the Rydberg state is unoccupied, there will be a significant mismatch. This fact can be easily seen from Eq. (C7). For example, in the occupied case, the $g_{m}^{2}$ term is much larger than the loss terms, so we can approximate

$$
\hat{b}_{\text {out }}(\omega) \approx \frac{i \omega+g_{m}^{2} /\left(i \omega-\gamma_{s}\right)}{i \omega+g_{m}^{2} /\left(i \omega-\gamma_{s}\right)} \hat{b}_{\text {in }}(\omega)=\hat{b}_{\text {in }}(\omega),
$$

which means $C_{1}=1$ and no mode function mismatch. When $g_{m}$ decreases and thereby $\kappa / g_{m}$ increases, the system will deviate from the strong-coupling region, and the wave-form mismatch $1-\Lambda$ (defined in the main text) becomes larger. This explains the feature of the solid curves in Fig. 4 of the main text.

However, when the Rydberg state is empty, $g_{m}=0$, and Eq. (C7) is

$$
\hat{b}_{\text {out }}(\omega)=\frac{i \omega+\kappa / 2}{i \omega-\kappa / 2} \hat{b}_{\text {in }}(\omega) \approx-e^{i 4 \omega / \kappa} \hat{b}_{\text {in }}(\omega),
$$

there will be a phase delay whose magnitude is proportional to $1 / \kappa$. When $\kappa$ increases, the mismatch will decrease, which explains the features of dashed lines in Fig. 4 of the main text.

\section{APPENDIX D: LINEARIZATION OF CQED DYNAMICS}

In the above derivation, we approximate the CQED dynamics by linearization. Having a linearized dynamics of the cQED system is also essential for a high-fidelity processing of the MW Schrödinger cat qubit; otherwise, the nonlinear dynamics of the $\mathrm{cQED}$ system will distort the MW Schrödinger cat qubit. In this Appendix two important points of this linear approximation are discussed.

Firstly, we discuss the validity conditions of this linearized dynamics: (1) the coherent amplitude $\alpha$ in the cat state cannot be too large (but also cannot be too small for applications in quantum information science, usually $\alpha^{2} \geqslant 2$ ); (2) strong-coupling region $g_{m}^{2} / \kappa \gamma_{s} \gg 1$. The errors of the fidelity calculated by linearized cQED dynamics and the simulation result without linearization have been shown in Fig. 7. It is clear that the validity of the linear approximation $\left\langle\hat{\sigma}_{z}\right\rangle \approx-1 / 2$ will be degraded by decreasing the coupling strength $g_{m}$ and increasing the cavity bandwidth $\kappa$ and the coherent amplitude $\alpha$. In the following we give have a detailed analysis of the validity of this linear approximation.

Our approach to examine the validity of linear approximation is to check the consistency of this approximation by substituting the result calculated under $\left\langle\hat{\sigma}_{z}\right\rangle \approx-1 / 2$ to the $\hat{\sigma}_{z}$ equation of motion:

$$
\dot{\hat{\sigma}}_{z}(t)=i g_{m} \hat{\sigma}_{r_{1} r_{2}}(t) \hat{a}_{c}^{\dagger}(t)+\text { H.c. }
$$

Here, by Fourier-transforming the result obtained in the Appendix C, we have

$\hat{\sigma}_{r_{1} r_{2}}(t)=\int \frac{i g_{m} \sqrt{\kappa} e^{-i \omega\left(t-t^{\prime}\right)}}{-\omega^{2}-i\left(\kappa / 2+\gamma_{s}\right) \omega+\kappa \gamma_{s} / 2+g_{m}^{2}} \hat{a}_{\text {in }}\left(t^{\prime}\right) d \omega d t^{\prime}$.

In our system we can neglect the $\omega^{2}$ term as an approximation since we have the long pulse as the initial state:

$$
\hat{\sigma}_{r_{1} r_{2}}(t)=\frac{i g_{m} \sqrt{\kappa}}{\left(\kappa / 2+\gamma_{s}\right)} \int \frac{e^{-i \omega\left(t-t^{\prime}\right)}}{-i \omega+\Gamma} \hat{a}_{\mathrm{in}}\left(t^{\prime}\right) d \omega d t^{\prime},
$$

with

$$
\Gamma=\frac{\kappa \gamma_{s} / 2+g_{m}^{2}}{\left(\kappa / 2+\gamma_{s}\right)} .
$$

Solving the integral leads to [where $\hat{\sigma}_{r_{1} r_{2}}(0)=0$ is assumed]

$$
\hat{\sigma}_{r_{1} r_{2}}(t)=\frac{i g_{m} \sqrt{\kappa}}{\gamma_{s}+\kappa / 2} \int_{-\infty}^{t} e^{-\Gamma\left(t-t^{\prime}\right)} \hat{a}_{\text {in }}\left(t^{\prime}\right) d t^{\prime} .
$$

The intracavity MW field $\hat{a}(t)$ is given by

$$
\begin{aligned}
\hat{a}_{c}(t)= & \int \frac{\sqrt{\kappa}\left(i \omega-\gamma_{s}\right) e^{-i \omega\left(t-t^{\prime}\right)}}{-\omega^{2}-i\left(\gamma_{s}+\kappa / 2\right) \omega+g_{m}^{2}+\kappa \gamma_{s} / 2} \hat{a}_{\text {in }}\left(t^{\prime}\right) d \omega d t^{\prime} \\
= & \sqrt{\kappa} \int_{-\infty}^{t} e^{-\left(\gamma_{s} / 2+\kappa / 4\right)\left(t-t^{\prime}\right)}\left[\eta \sin \chi\left(t-t^{\prime}\right)\right. \\
& \left.-\cos \chi\left(t-t^{\prime}\right)\right] \hat{a}_{\text {in }}\left(t^{\prime}\right) d t^{\prime},
\end{aligned}
$$

with

$$
\begin{aligned}
& \chi=\sqrt{-\left(\gamma_{s}+\kappa / 2\right)^{2}+4\left(g_{m}^{2}+\kappa \gamma_{s} / 2\right)} / 2, \\
& \eta=\left(\kappa / 2-\gamma_{s}\right) / \sqrt{-\left(\gamma_{s}+\kappa / 2\right)^{2}+4\left(g_{m}^{2}+\kappa \gamma_{s} / 2\right)} .
\end{aligned}
$$

For an orders-of-magnitude estimation, let us assume that the input field is a steady coherent field $|\alpha\rangle$. Then the above two integrals can be explicitly calculated and we have

$$
\left\langle\hat{\sigma}_{r_{1} r_{2}}\right\rangle=\frac{i g_{m} \sqrt{\kappa} \alpha}{\left(\gamma_{s}+\kappa / 2\right) \Gamma}, \quad\left\langle\hat{a}_{c}\right\rangle=\frac{-2 \sqrt{\kappa} \alpha \gamma_{s}}{2 g_{m}^{2}+\kappa \gamma_{s}} .
$$



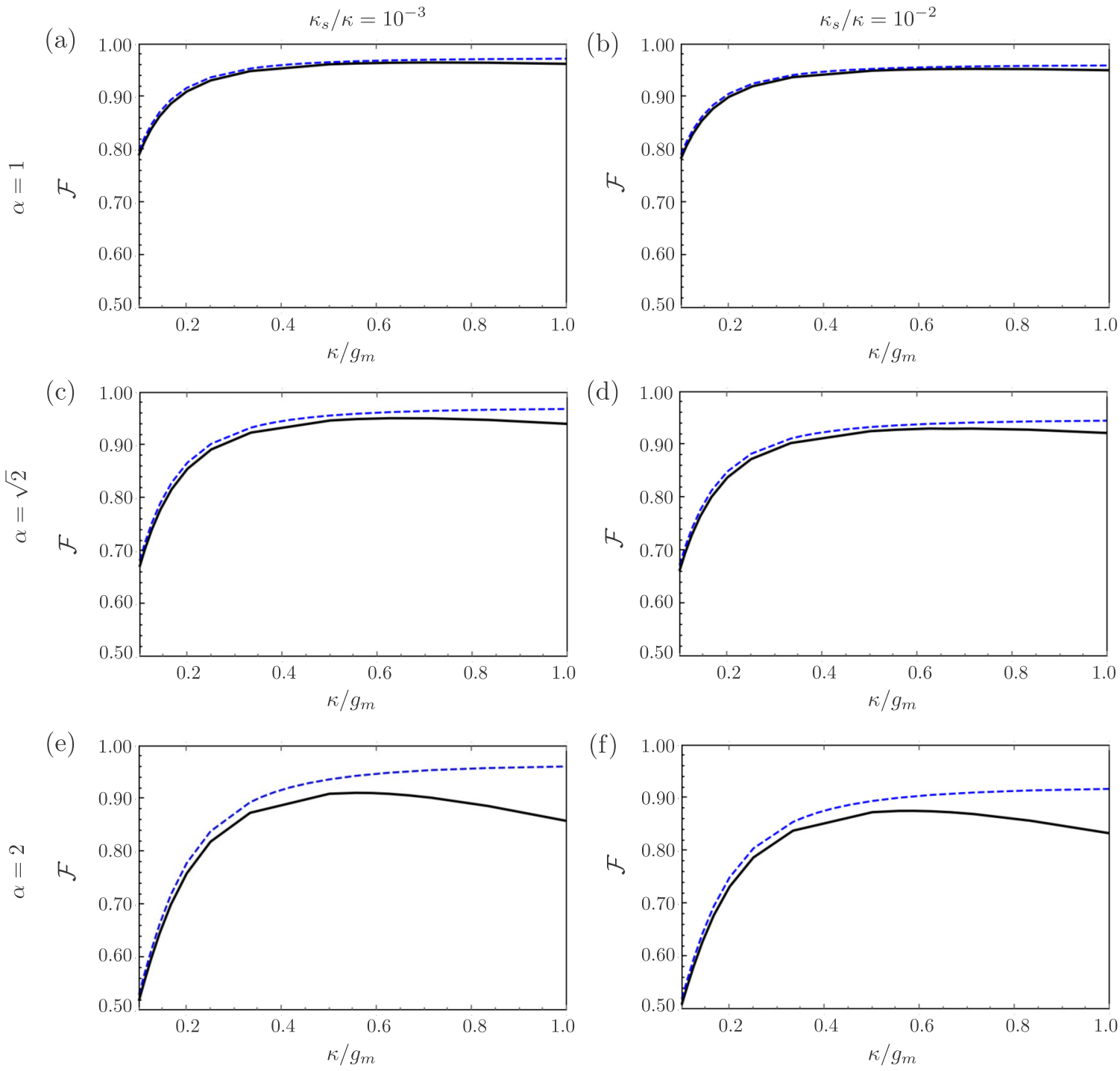

FIG. 7. The errors of fidelity calculation using the linear approximation $\left\langle\hat{\sigma}_{z}\right\rangle \approx-1 / 2$. The dashed and solid curve are the results under linear approximation, and the results obtained by full simulations, respectively. The left/right panel corresponds to $\kappa_{s}=0.001 \kappa$ and $\kappa_{s}=$ $0.01 \kappa$, respectively. The three rows correspond to $\alpha=1[(\mathrm{a}),(\mathrm{b})], \sqrt{2}[(\mathrm{c}),(\mathrm{d})]$, and $2[(\mathrm{e}),(\mathrm{f})]$, respectively. It is clear that the error increases with the increasing of $\kappa / g_{m}$ and $\alpha$, which is well consistent with our theoretical analysis.

It is clear that cavity field is very small for typical parameters; this reflects the fact that when the Rydberg state is occupied, the MW fields feel a reflection almost without phase delay. Then, to leading order, the correction to $\left\langle\hat{\sigma}_{z}\right\rangle \approx-1 / 2$ is given by

$$
\left\langle\delta \dot{\hat{\sigma}}_{z}\right\rangle=\frac{2 \kappa g_{m}^{2} \gamma_{s}}{\left(g_{m}^{2}+\kappa \gamma_{s} / 2\right)^{2}} \alpha_{\mathrm{in}}^{2} \approx \frac{2 \kappa \gamma_{s}}{g_{m}^{2}} \alpha_{\mathrm{in}}^{2} .
$$

Clearly, when we have large enough "cooperativity" $g_{m}^{2} / \kappa \gamma_{s}$ and small enough $\alpha$, the error to the linear approximation can be tiny. This result has a transparent physical explanation, since larger cooperativity means a stronger Purcell effect, which tends to deexcite the atom state $\left|r_{2}\right\rangle$.

Secondly, we discuss the physical scenarios of this linearized dynamics. For example, when the input optical qubit is $\left(\left|1_{R}\right\rangle+\left|1_{L}\right\rangle\right) / \sqrt{2}$, the spin wave can be recorded in the superposition of $\left|r_{1}\right\rangle-|g\rangle$ and $\left|g^{\prime}\right\rangle-|g\rangle$. This means that the
Rydberg state is either being occupied at $\left|r_{1}\right\rangle$ or simply empty. Therefore, as long as the small $\alpha$ and large cooperativity conditions are satisfied, the linear approximation $\left\langle\hat{\sigma}_{z}\right\rangle=-1 / 2$ can be applied to both of these two situations. As an example, suppose we have joint input state $|\psi\rangle=\left(\left|1_{R}\right\rangle+\left|1_{L}\right\rangle\right) / \sqrt{2} \otimes$ $|\alpha\rangle$. Then the evolution can be written (in the lossless case) as

$$
\begin{aligned}
\hat{U}|\psi\rangle= & \frac{1}{\sqrt{2}}\left|1_{R}\right\rangle \otimes \hat{U} \exp \left[\alpha \int d x f_{\text {in }}(x) \hat{b}_{\mathrm{in}}^{\dagger}(x)\right]|0\rangle \\
& +\frac{1}{\sqrt{2}}\left|1_{L}\right\rangle \otimes \hat{U} \exp \left[\alpha \int d x f_{\mathrm{in}}(x) \hat{b}_{\mathrm{in}}^{\dagger}(x)\right]|0\rangle \\
= & \frac{1}{\sqrt{2}}\left|1_{R}\right\rangle \otimes \exp \left[\alpha \int d x f_{\mathrm{in}}(x) \hat{b}_{\mathrm{out}}^{\dagger g_{m} \neq 0}(x)\right]|0\rangle \\
& +\frac{1}{\sqrt{2}}\left|1_{L}\right\rangle \otimes \exp \left[\alpha \int d x f_{\mathrm{in}}(x) \hat{b}_{\mathrm{out}}^{\dagger g_{m}=0}\right]|0\rangle,
\end{aligned}
$$


where $\hat{b}_{\text {out }}^{g_{m}=/ \neq 0}$ is given in Eq. (C6), where $g_{m}=0$ and $g_{m} \neq 0$ correspond to the empty or occupied cavity, respectively. This method is used in computing the fidelity of our system.

\section{APPENDIX E: FIDELITY}

For those transformations in the truth table of which no entanglement was generated, the gate fidelity can be simply calculated as $\mathscr{F}=\mathscr{F}_{\mathrm{MW}} \times \mathscr{F}_{\text {opt }}$, and the result has been shown in the truth table in the main text. However, a truth table can be simulated by the classical computer. For a quantum $\mathrm{CZ}$ gate with the gate matrix

$$
\hat{U}_{\mathrm{CZ}}=\left[\begin{array}{cccc}
1 & 0 & 0 & 0 \\
0 & 1 & 0 & 0 \\
0 & 0 & 1 & 0 \\
0 & 0 & 0 & -1
\end{array}\right],
$$

there exists transformations which generate optical-MW qubit entanglement. For example,

$$
\begin{aligned}
& \left.\left.\hat{U}_{\mathrm{CZ}}\left[\left(|1\rangle_{L}+|1\rangle_{R}\right) \otimes(\mid \text { even }\rangle+\mid \text { odd }\right\rangle\right)\right] \\
& \left.\left.\left.\left.\quad=|1\rangle_{L} \otimes(\mid \text { even }\rangle-\mid \text { odd }\right\rangle\right)+|1\rangle_{\mathrm{R}} \otimes(\mid \text { even }\rangle+\mid \text { odd }\right\rangle\right) .
\end{aligned}
$$

Therefore it is of crucial importance to consider the fidelity of these entanglement generation processes. We plot the resultant fidelity in Fig. 8 for two different MW cavity losses $\kappa_{s} / \kappa=0.01,0.001$ and different coherent amplitudes of the Schrödinger cat state $\alpha=(1, \sqrt{2}, 2)$, where we use the rotation operators on the Bloch sphere (see the main text) to represent different optical and MW input states (see Fig. 8). The operation of the quantum $\mathrm{CZ}$ gate on the separable input states can produce entangled states. In the following we will briefly discuss the way we compute the fidelity.

The separable input qubit state is

$$
\left.\left.|\psi\rangle_{q}^{\text {in }}=\left(c_{1}|1\rangle_{R}+c_{2}|1\rangle_{L}\right) \otimes\left(c_{3} \mid \text { even }\right\rangle+c_{4} \mid \text { odd }\right\rangle\right),
$$

where

$$
\begin{aligned}
\mid \text { even }\rangle & =\frac{1}{\sqrt{2+e^{-2 \alpha^{2}}}}(|\alpha\rangle+|-\alpha\rangle), \\
\mid \text { odd }\rangle & =\frac{1}{\sqrt{2-e^{-2 \alpha^{2}}}}(|\alpha\rangle-|-\alpha\rangle) .
\end{aligned}
$$

Then the output state after the postselection is

$$
\begin{aligned}
|\Psi\rangle^{\text {out }}= & A_{+} C_{1}^{o R}\left|1_{\text {outR }}\right\rangle_{1}\left|\alpha_{\text {outR }}\right\rangle \otimes|g\rangle\left|E_{\alpha \mathrm{R}}\right\rangle \\
& +A_{-} C_{1}^{o R}\left|1_{\text {outR }}\right\rangle_{1}\left|-\alpha_{\text {outR }}\right\rangle \otimes|g\rangle\left|E_{-\alpha \mathrm{R}}\right\rangle \\
& +B_{+} C_{1}^{o L}\left|1_{\text {outL }}\right\rangle_{1}\left|\alpha_{\text {outL }}\right\rangle \otimes|g\rangle\left|E_{\alpha \mathrm{L}}\right\rangle \\
& +B_{-} C_{1}^{o L}\left|1_{\text {outL }}\right\rangle_{1}\left|-\alpha_{\text {outL }}\right\rangle \otimes|g\rangle\left|E_{-\alpha \mathrm{L}}\right\rangle
\end{aligned}
$$

where we have omitted those terms where the optical qubit is lost to the environment and

$$
\begin{aligned}
& A_{ \pm}=\frac{c_{1} c_{3}}{\sqrt{2+e^{-2 \alpha^{2}}}} \pm \frac{c_{1} c_{4}}{\sqrt{2-e^{-2 \alpha^{2}}}}, \\
& B_{ \pm}=\frac{c_{2} c_{3}}{\sqrt{2+e^{-2 \alpha^{2}}}} \pm \frac{c_{2} c_{4}}{\sqrt{2-e^{-2 \alpha^{2}}}} .
\end{aligned}
$$

The ideal output field is given by

$$
|\psi\rangle_{q}^{\text {ideal }}=\sum_{ \pm} A_{ \pm}|1\rangle_{R} \otimes| \pm \alpha\rangle+B_{ \pm}|1\rangle_{L} \otimes| \pm \alpha\rangle
$$

The output qubit density matrix after postselection is

$$
\rho_{q}^{\text {out }}=\operatorname{Tr}_{g, E}\left[|\Psi\rangle^{\text {out }}\left\langle\left.\Psi\right|^{\text {out }}\right],\right.
$$

where we trace out the atom and environmental degrees of freedom. Thus the fidelity is given by

$$
\mathscr{F}=\operatorname{Tr}\left[\hat{\rho}_{q}^{\text {out }} \hat{\rho}_{\text {ideal }}\right] \text {. }
$$

The result is quite cumbersome and can be expressed as

$$
\begin{aligned}
\mathscr{F}= & \sum_{i j, m n}^{a b, c d} f_{i j} f_{m n} f_{a b} f_{c d}\left\langle E_{m n} \mid E_{i j}\right\rangle\left\langle a_{\text {out }} \mid 1_{\text {out } i}\right\rangle\left\langle 1_{\text {out } m} \mid c_{\text {out }}\right\rangle \\
& \left\langle(-1)^{a} b_{\text {out }} \mid(-1)^{i} 1_{\text {out } j}\right\rangle\left\langle(-1)^{m} 1_{\text {out } n} \mid(-1)^{c} d_{\text {out }}\right\rangle,
\end{aligned}
$$

where the indices $(i, m, a, c)$ represent the polarizations $(R, L)$ and $(j, n, b, d)$ represent $(\alpha,-\alpha)$, and we define $(-1)^{L / R}=$ $(-/+) 1$. The $f$ coefficient is the matrix element of $\mathbb{F}$ :

$$
\mathbb{F}=\left(\begin{array}{l}
A_{+} \\
A_{-} \\
B_{+} \\
B_{-}
\end{array}\right) \otimes\left(\begin{array}{llll}
A_{+}^{*} & A_{-}^{*} & B_{+}^{*} & B_{-}^{*}
\end{array}\right),
$$

where the row/column order is $(R, L, \alpha,-\alpha)$.

In computing the above fidelity, we need to use the following inner product of the MW environment states:

$$
\left\langle E_{\alpha i} \mid E_{-\alpha j}\right\rangle=\exp \left[-\alpha^{2} \int d \omega\left|f_{\text {in }}(\omega)\right|^{2} C_{2 j}^{*}(\omega) C_{2 i}(\omega)\right],
$$

where $C_{2 i}(\omega)$ is given by Eq. (C7) with $g_{m}=/ \neq 0$ when $i=$ $L / R$, respectively.

As a special case, the fidelity for the truth table can be easily calculated as follows. The optical part $\mathscr{F}_{\text {opt }}$, with the ideal output field

$$
|\Psi\rangle_{\text {out }}^{\text {ideal }}=\int d k f_{\text {ideal }}(k) \hat{\epsilon}_{k}^{\dagger}(0)|\mathrm{vac}\rangle,
$$

can be written as

$$
\begin{aligned}
\mathscr{F}_{\text {opt }} & =\left|\left\langle\Psi_{\text {out }}^{\text {ideal }} \mid \Psi_{\text {out }}\right\rangle\right|^{2} \\
& =\int d k f_{\text {ideal }}^{*}\left(k^{\prime}\right) f_{\text {in }}(k, T) \chi^{*}(k) e^{-i \Phi_{T}(k)} .
\end{aligned}
$$

The MW part, $\mathscr{F}_{\text {MW }}$, is given by

$$
\begin{aligned}
\mathscr{F} & =\left\langle\left.\Psi\right|_{\text {in }} ^{\mathrm{MW}} \hat{\rho}_{\text {out }}^{q} \mid \Psi\right\rangle_{\text {in }}^{\mathrm{MW}} \\
& =4 \mathscr{N}(1+\xi)\left(\cos 2 \alpha^{2} \Lambda_{i}+\cosh 2 \alpha^{2} \Lambda_{r}\right),
\end{aligned}
$$

where we have

$$
\begin{aligned}
& \Lambda=\int d \omega\left|f_{\text {in }}(\omega)\right|^{2} C_{1}^{*}(\omega)=\Lambda_{r}+i \Lambda_{i}, \\
& \xi=\left\langle E_{\alpha} \mid E_{-\alpha}\right\rangle=\exp \left[-\alpha^{2} \int d \omega\left|f_{\text {in }}(\omega) C_{2}(\omega)\right|^{2}\right]
\end{aligned}
$$

and the normalization factor

$$
\mathscr{N}=\exp \left[-2 \alpha_{0}^{2}\right] /\left(2 \pm \exp \left[-2 \alpha_{0}^{2}\right]\right)^{2} \text {. }
$$

The final fidelity for the truth table is simply $\mathscr{F}=\mathscr{F}_{\text {MW }} \times$ $\mathscr{F}_{\text {opt }}$. 


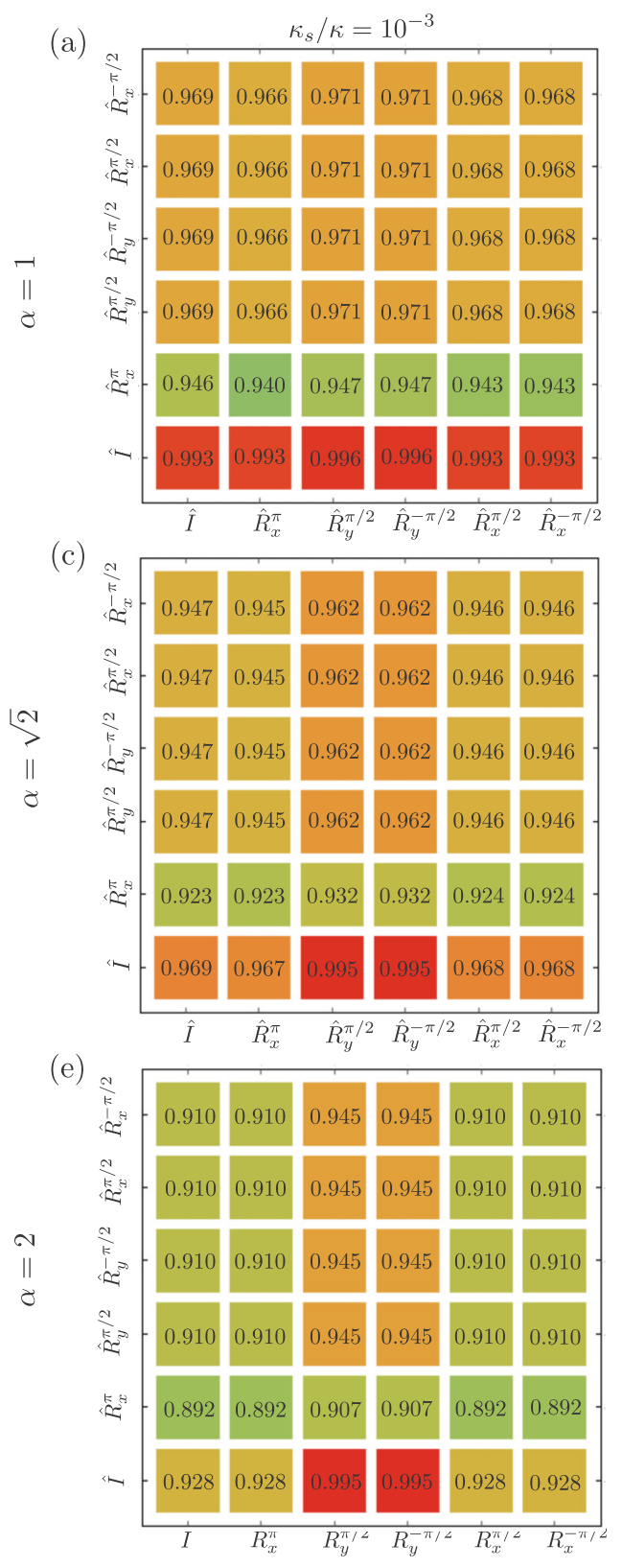

(b)

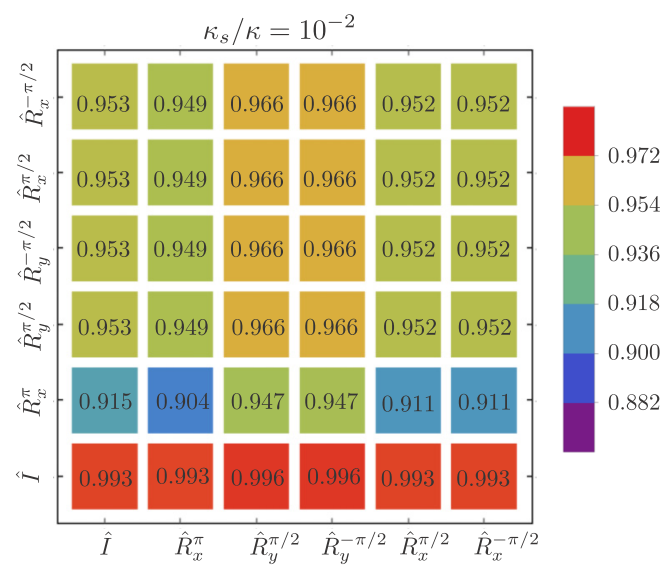

(d)

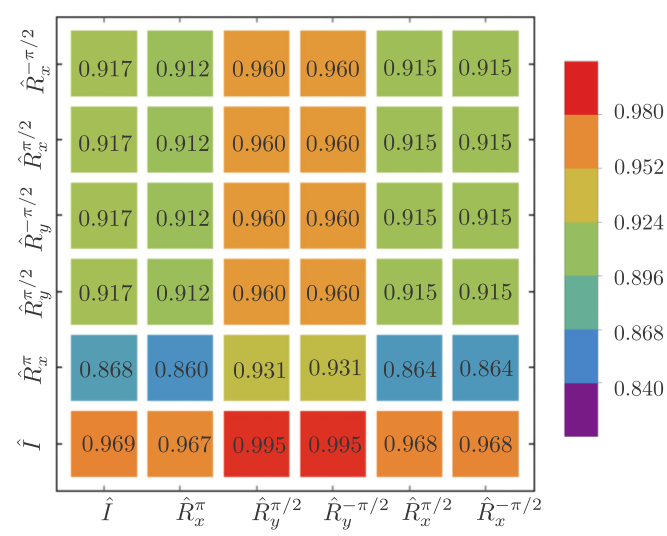

(f)

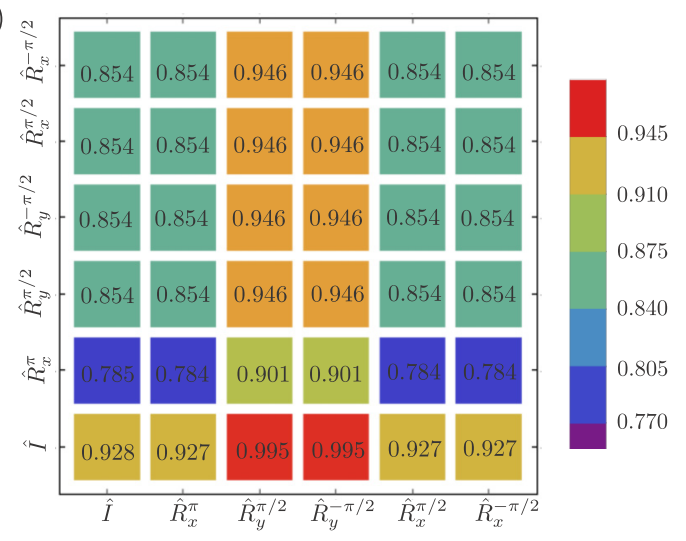

FIG. 8. The fidelity of the hybrid quantum gate for the $\mathrm{CZ}$ gate with separable input states $|\psi\rangle_{q}^{o} \otimes|\psi\rangle_{q}^{\mathrm{MW}}$ when $\kappa_{s}=10^{-3} \kappa[(\mathrm{a}),(\mathrm{c})$, (e)] and $\kappa_{s}=10^{-2} \kappa[(\mathrm{b}),(\mathrm{d}),(\mathrm{f})]$. The vertical and horizontal axis represent optical qubit states $|\psi\rangle_{q}^{o}$ and the MW qubit states $|\psi\rangle_{q}^{\mathrm{MW}}$, respectively. The color represent the fidelity values. For illustrative purposes, we chose a fiducial state as $\left|\psi_{\mathrm{FD}}\right\rangle=|1\rangle_{o}^{R}$ or $\mid$ even $\rangle$, and other states are represented by operations on the Bloch sphere (see the main text) $\hat{R}_{j}^{\theta}$, where $j$ is the axis index and $\theta$ is the rotation angle. The concrete fidelity value of each input state is also marked.

\section{APPENDIX F: PARAMETER SETTING}

Sampling parameters. In choosing parameters, the strong interaction strength of the cavity QED system leads to some constraints to other system parameters. The Rabi frequency of cQED systems driven by a single photon in continuous mode is given by

$$
g_{m}=\sqrt{\frac{\hbar \omega_{c}}{2 \epsilon_{0} V_{c}}} \frac{p_{r_{1} r_{2}}}{\hbar} u(r),
$$

where $p_{r_{1} r_{2}}$ is the Rydberg dipole transition amplitude, and $u(r)$ is the cross-sectional field distribution. For the system to work in the strong-coupling region where $g_{m} / \kappa \gg 1$, the cavity bandwidth $\kappa$ cannot be too large, which means the cavity damping time cannot be too short. At the same time, for a high-fidelity gate, the lifetime of the atom state $\left|r_{1}\right\rangle$ cannot be shorter than the cavity damping time, that is, $\gamma_{r_{1} g}<\kappa \ll g_{m}$ is required.

Reducing the temperature of the atomic ensemble can lead to a small $\gamma_{r_{1} g}$, since the thermal Doppler effect of atoms mainly contributes to $\gamma_{r_{1} g}$ as $\gamma_{r_{1} g} \approx\left(k_{g e}-k_{e r_{1}}\right) k_{B} T / m$. Since the single-photon optical qubit at most excites only one atom to the Rydberg state, therefore the collective effect discussed in [75] has no enhancement to $\gamma_{r_{1} g}$. If we bring the temperature 
(a)

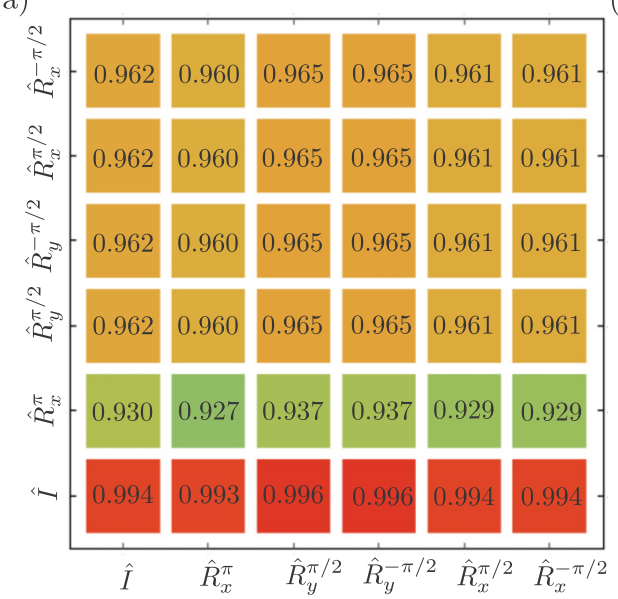

(c)

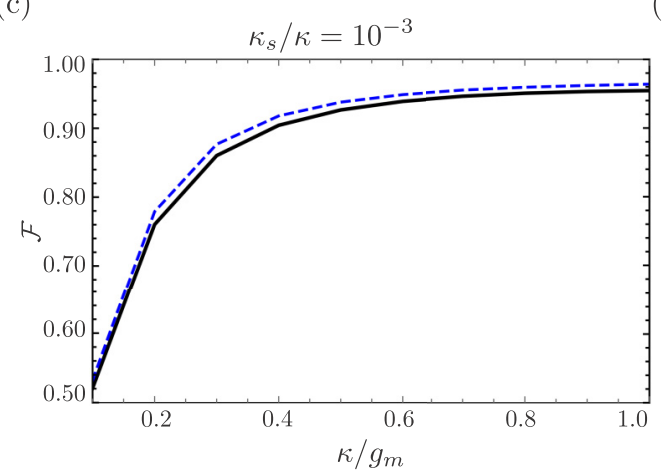

(b)

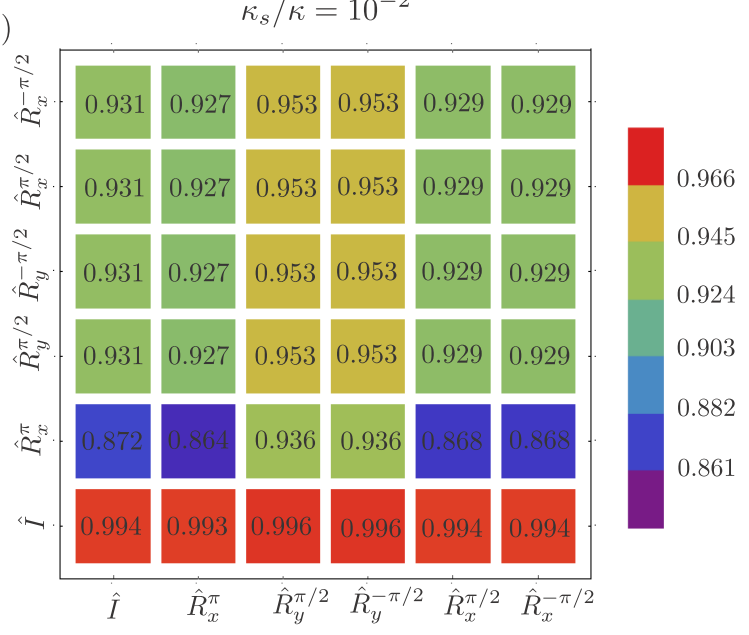

(d)

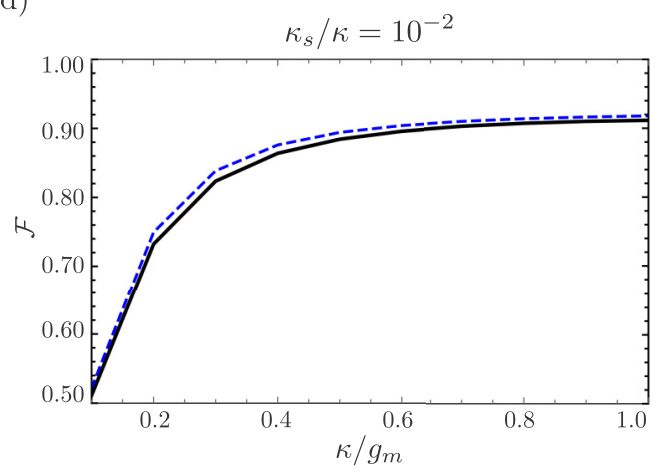

FIG. 9. Upper plane (a), (b): The fidelity of the hybrid quantum gate for CZ gate with separable input states $|\psi\rangle_{q}^{o} \otimes|\psi\rangle_{q}^{\mathrm{MW}}$ for the sampling parameters when $n$ is around 40 and for $\alpha=2$. The fidelity is apparently improved compared to the results shown in Fig. 8 due to less deviation from linear cQED dynamics. In this case, the Schrödinger cat state $\alpha=2$ can also achieve a high gate fidelity. The dashed and solid curve are the results under linear approximation and the results obtained by full simulations as shown, (c) and (d), respectively.

down to $0.2 \mu \mathrm{K}$, we will get $\gamma_{r_{1} g}=2 \pi \times 3.5 \mathrm{kHz}$. Previous work for achieving the strong electric dipole moment of the Rydberg atom proposed to use a MW resonator based on superconducting circuits $[34,39,68]$. This method could also be useful for our proposal. We assume that the grounded superconducting electrodes locate at distance $w_{c}=25 \mu \mathrm{m}$, which confines the cavity field within the effective volume $V_{c}=\int \mathrm{d}^{3} r|u(r)|^{2} \approx \frac{\pi}{2} \omega^{2} L$ and the cavity length is taken to be $L=0.45 \mathrm{~cm}$. The relevant transition dipole matrix element from $\left|r_{1}\right\rangle$ to $\left|r_{2}\right\rangle$ is $1898 a_{0} e$. We also set the distance between the atom ensemble and the coplanar superconducting circuit surface as determined by the mode function equation $u(r) \approx$ $e^{-1}$. Then we can obtain $g_{m}=2 \pi \times 2.723 \mathrm{MHz}$. Actually, the $g_{m}$ can be even larger as discussed in [68]. In Fig. 8 it is clear that the fidelity depends on the value of $\kappa_{s}$. For coplanar superconducting resonators, recent state-of-the-art methods can achieve the loss level to $\kappa_{s} / \kappa=0.001$ (i.e., the internal quality factor to be $10^{6}-10^{7}$ ), where the loss is dominated by the interfacial and surface two-level states (the so-called TLS loss) [72].

Scaling with principle quantum number and further discussions. In the main text, as an example the simulation is performed for the system where the principle quantum number for the Rydberg state is chosen to be $n=69$. This case corresponds to the $10-\mathrm{GHz} \mathrm{MW}$ frequency, which is widely used in MW quantum optics. Here we further investigate the dependence of the system performance on $n$. The scaling of parameters of $n$ in Eq. (F1) can be summarized as follows. The dipole moments $p_{r_{1} r_{2}} \propto n^{2}$, and the resonant frequency $\omega_{c} \propto 1 / \lambda \propto n^{-3}$. The volume $V_{c} \propto A \lambda \propto A n^{3}$, where $A$ is the cross-sectional area of the MW field. Therefore we can conclude that $g_{m} \propto 1 / n$, which means the interaction strength can be increased by decreasing $n$.

There are some advantages of using a Rydberg state with smaller $n$. (1) A Rydberg state with smaller $n$ has a smaller dipole moment, which could reduce the decoherence rate due to the interaction with stray electric fields. (2) Smaller $n$ and a proper choice of the $l$ can increase the coupling strength $g_{m}$ so that the linear QED dynamics can be preserved for a Schrödinger cat state with higher $\alpha$, which is very important for future quantum information processing. As an example, we have computed the case with the amplitude of the MW Schrödinger cat state to be $\alpha=2$ and the Rydberg states are $\left|r_{1}\right\rangle=\mid 40 D, F=m_{F}=$ $4\rangle,\left|r_{2}\right\rangle=\left|39 F, F=m_{F}=5\right\rangle$ with $g_{m}=2 \pi \times 6 \mathrm{MHz}$; the result is shown in Fig. 9. It is clear that the fidelity improves compared to $\alpha=2$ in Fig. 8, and the cQED dynamics driven by the $\alpha=2$ Schrödinger cat state is much less deviated from the linear approximation due to larger $g_{m}$. 
Finally, we want to emphasize here that our theoretical analysis is to reveal the physical features and potential of our protocol, while the final experimental realization needs more complete and realistic considerations.
[1] M. Pompili, S. L. N. Hermans, S. Baier, H. K. C. Beukers, P. C. Humphreys, R. N. Schouten, R. F. L. Vermeulen, M. J. Tiggelman, L. dos Santos Martins, B. Dirkse, S. Wehner, and R. Hanson, Realization of a multinode quantum network of remote solid-state qubits, Science 372, 259 (2021).

[2] S. Daiss, S. Langenfeld, S. Welte, E. Distante, P. Thomas, L. Hartung, O. Morin, and G. Rempe, A quantum-logic gate between distant quantum-network modules, Science 371, 614 (2021).

[3] C.-W. Chou, J. Laurat, H. Deng, K. S. Choi, H. de Riedmatten, D. Felinto, and H. J. Kimble, Functional quantum nodes for entanglement distribution over scalable quantum networks, Science 316, 1316 (2007).

[4] J. I. Cirac, P. Zoller, H. J. Kimble, and H. Mabuchi, Quantum State Transfer and Entanglement Distribution among Distant Nodes in a Quantum Network, Phys. Rev. Lett. 78, 3221 (1997).

[5] L.-M. Duan, M. D. Lukin, J. I. Cirac, and P. Zoller, Longdistance quantum communication with atomic ensembles and linear optics, Nature (London) 414, 413 (2001).

[6] K. Stannigel, P. Rabl, A. S. Sørensen, P. Zoller, and M. D. Lukin, Optomechanical Transducers for Long-Distance Quantum Communication, Phys. Rev. Lett. 105, 220501 (2010).

[7] S. Muralidharan, L. Li, J. Kim, N. Lütkenhaus, M. D. Lukin, and L. Jiang, Optimal architectures for long distance quantum communication, Sci. Rep. 6, 20463 (2016).

[8] J. Wallnöfer, A. A. Melnikov, W. Dür, and H. J. Briegel, Machine learning for long-distance quantum communication, PRX Quantum 1, 010301 (2020).

[9] F. Zhu, W. Zhang, Y. Sheng, and Y. Huang, Experimental longdistance quantum secure direct communication, Sci. Bull. 62, 1519 (2017).

[10] I. Cohen and K. Mølmer, Deterministic quantum network for distributed entanglement and quantum computation, Phys. Rev. A 98, 030302(R) (2018).

[11] D. Cuomo, M. Caleffi, and A. S. Cacciapuoti, Towards a distributed quantum computing ecosystem, IET Quantum Commun. 1, 3 (2020).

[12] R. Van Meter and S. J. Devitt, The path to scalable distributed quantum computing, Computer 49, 31 (2016).

[13] R. Beals, S. Brierley, O. Gray, A. W. Harrow, S. Kutin, N. Linden, D. Shepherd, and M. Stather, Efficient distributed quantum computing, Proc. R. Soc. London, Ser. A 469, 20120686 (2013).

[14] Y. L. Lim, A. Beige, and L. C. Kwek, Repeat-Until-Success Linear Optics Distributed Quantum Computing, Phys. Rev. Lett. 95, 030505 (2005).

[15] H. Zhou, J. Choi, S. Choi, R. Landig, A. M. Douglas, J. Isoya, F. Jelezko, S. Onoda, H. Sumiya, P. Cappellaro, H. S. Knowles, H. Park, and M. D. Lukin, Quantum Metrology with Strongly Interacting Spin Systems, Phys. Rev. X 10, 031003 (2020).

[16] V. Giovannetti, S. Lloyd, and L. Maccone, Quantum Metrology, Phys. Rev. Lett. 96, 010401 (2006).

[17] V. Giovannetti, S. Lloyd, and L. Maccone, Advances in quantum metrology, Nat. Photonics 5, 222 (2011).
[18] G. Tóth and I. Apellaniz, Quantum metrology from a quantum information science perspective, J. Phys. A: Math. Theor. 47, 424006 (2014).

[19] A. W. Chin, S. F. Huelga, and M. B. Plenio, Quantum Metrology in Non-Markovian Environments, Phys. Rev. Lett. 109, 233601 (2012).

[20] J. Joo, W. J. Munro, and T. P. Spiller, Quantum Metrology with Entangled Coherent States, Phys. Rev. Lett. 107, 083601 (2011).

[21] M. Kjaergaard, M. E. Schwartz, J. Braumüller, P. Krantz, J. I.J. Wang, S. Gustavsson, and W. D. Oliver, Superconducting qubits: Current state of play, Annu. Rev. Condens. Matter Phys. 11, 369 (2020).

[22] Y.-A. Chen, Q. Zhang, T.-Y. Chen, W.-Q. Cai, S.-K. Liao, J. Zhang, K. Chen, J. Yin, J.-G. Ren, Z. Chen, S.-L. Han, Q. Yu, K. Liang, F. Zhou, X. Yuan, M.-S. Zhao, T.-Y. Wang, X. Jiang, L. Zhang et al., An integrated space-to-ground quantum communication network over 4,600 kilometres, Nature (London) 589, 214 (2021).

[23] F. Arute, K. Arya, R. Babbush, D. Bacon, J. Bardin, R. Barends, R. Biswas, S. Boixo, F. Brandao, D. Buell, B. Burkett, Y. Chen, Z. Chen, B. Chiaro, R. Collins, W. Courtney, A. Dunsworth, E. Farhi, B. Foxen, and J. Martinis, Quantum supremacy using a programmable superconducting processor, Nature (London) 574, 505 (2019).

[24] R. Barends, J. Kelly, A. Megrant, A. Veitia, D. Sank, E. Jeffrey, T. White, J. Mutus, A. Fowler, B. Campbell, Y. Chen, Z. Chen, B. Chiaro, A. Dunsworth, C. Neill, P. O’Malley, P. Roushan, A. Vainsencher, J. Wenner, and J. Martinis, Superconducting quantum circuits at the surface code threshold for fault tolerance, Nature (London) 508, 500 (2014).

[25] G. Wendin, Quantum information processing with superconducting circuits: A review, Rep. Prog. Phys. 80, 106001 (2017).

[26] S. Slussarenko and G. J. Pryde, Photonic quantum information processing: A concise review, Appl. Phys. Rev. 6, 041303 (2019).

[27] P. Kok, W. J. Munro, K. Nemoto, T. C. Ralph, J. P. Dowling, and G. J. Milburn, Linear optical quantum computing with photonic qubits, Rev. Mod. Phys. 79, 135 (2007).

[28] G. A. Peterson, F. Lecocq, K. Cicak, R. W. Simmonds, J. Aumentado, and J. D. Teufel, Demonstration of Efficient Nonreciprocity in a Microwave Optomechanical Circuit, Phys. Rev. X 7, 031001 (2017).

[29] J. P. Covey, A. Sipahigil, and M. Saffman, Microwave-tooptical conversion via four-wave mixing in a cold ytterbium ensemble, Phys. Rev. A 100, 012307 (2019).

[30] C. Hermann-Avigliano, R. C. Teixeira, T. L. Nguyen, T. CantatMoltrecht, G. Nogues, I. Dotsenko, S. Gleyzes, J. M. Raimond, S. Haroche, and M. Brune, Long coherence times for Rydberg qubits on a superconducting atom chip, Phys. Rev. A 90, 040502(R) (2014).

[31] S. D. Hogan, J. A. Agner, F. Merkt, T. Thiele, S. Filipp, and A. Wallraff, Driving Rydberg-Rydberg Transitions from a 
Coplanar Microwave Waveguide, Phys. Rev. Lett. 108, 063004 (2012).

[32] A. Clerk, K. Lehnert, P. Bertet, J. Petta, and Y. Nakamura, Hybrid quantum systems with circuit quantum electrodynamics, Nat. Phys. 16, 257 (2020).

[33] G. Kurizki, P. Bertet, Y. Kubo, K. Mølmer, D. Petrosyan, P. Rabl, and J. Schmiedmayer, Quantum technologies with hybrid systems, Proc. Natl. Acad. Sci. USA 112 (13), 3866 (2015).

[34] J. D. Pritchard, J. A. Isaacs, M. A. Beck, R. McDermott, and M. Saffman, Hybrid atom-photon quantum gate in a superconducting microwave resonator, Phys. Rev. A 89, 010301(R) (2014).

[35] R. Schoelkopf and S. Girvin, Wiring up quantum systems, Nature (London) 451, 664 (2008).

[36] D. Yu, A. Landra, M. M. Valado, C. Hufnagel, L. C. Kwek, L. Amico, and R. Dumke, Superconducting resonator and Rydberg atom hybrid system in the strong coupling regime, Phys. Rev. A 94, 062301 (2016).

[37] Z.-L. Xiang, S. Ashhab, J. Q. You, and F. Nori, Hybrid quantum circuits: Superconducting circuits interacting with other quantum systems, Rev. Mod. Phys. 85, 623 (2013).

[38] M. Wallquist, K. Hammerer, P. Rabl, M. Lukin, and P. Zoller, Hybrid quantum devices and quantum engineering, Phys. Scr. 2009, 014001 (2009).

[39] A. A. Morgan and S. D. Hogan, Coupling Rydberg Atoms to Microwave Fields in a Superconducting Coplanar Waveguide Resonator, Phys. Rev. Lett. 124, 193604 (2020).

[40] B. He, A. V. Sharypov, J. Sheng, C. Simon, and M. Xiao, Two-Photon Dynamics in Coherent Rydberg Atomic Ensemble, Phys. Rev. Lett. 112, 133606 (2014).

[41] M. Khazali, C. R. Murray, and T. Pohl, Polariton Exchange Interactions in Multichannel Optical Networks, Phys. Rev. Lett. 123, 113605 (2019).

[42] D. Tiarks, S. Schmidt-Eberle, T. Stolz, G. Rempe, and S. Dürr, A photon-photon quantum gate based on Rydberg interactions, Nat. Phys. 15, 124 (2019).

[43] M. Khazali, K. Heshami, and C. Simon, Photon-photon gate via the interaction between two collective Rydberg excitations, Phys. Rev. A 91, 030301(R) (2015).

[44] B. Hacker, S. Welte, G. Rempe, and S. Ritter, A photon quantum gate based on a single atom in an optical resonator, Nature (London) 536, 193 (2016).

[45] A. V. Gorshkov, J. Otterbach, M. Fleischhauer, T. Pohl, and M. D. Lukin, Photon-Photon Interactions via Rydberg Blockade, Phys. Rev. Lett. 107, 133602 (2011).

[46] D. Paredes-Barato and C. S. Adams, All-Optical Quantum Information Processing Using Rydberg Gates, Phys. Rev. Lett. 112, 040501 (2014).

[47] S. Das, A. Grankin, I. Iakoupov, E. Brion, J. Borregaard, R. Boddeda, I. Usmani, A. Ourjoumtsev, P. Grangier, and A. S. Sørensen, Photonic controlled-phase gates through Rydberg blockade in optical cavities, Phys. Rev. A 93, 040303(R) (2016).

[48] Y. M. Hao, G. W. Lin, K. Xia, X. M. Lin, Y. P. Niu, and S. Q. Gong, Quantum controlled-phase-flip gate between a flying optical photon and a Rydberg atomic ensemble, Sci. Rep. 5, 10005 (2015).

[49] M. Saffman, T. G. Walker, and K. Mølmer, Quantum information with Rydberg atoms, Rev. Mod. Phys. 82, 2313 (2010).

[50] Y. Sun and P.-X. Chen, Analysis of atom-photon quantum interface with intracavity Rydberg-blocked atomic ensemble via two-photon transition, Optica 5, 1492 (2018).

[51] O. Lahad and O. Firstenberg, Induced Cavities for Photonic Quantum Gates, Phys. Rev. Lett. 119, 113601 (2017).

[52] J.-C. Besse, S. Gasparinetti, M. C. Collodo, T. Walter, P. Kurpiers, M. Pechal, C. Eichler, and A. Wallraff, Single-Shot Quantum Nondemolition Detection of Individual Itinerant Microwave Photons, Phys. Rev. X 8, 021003 (2018).

[53] S. Kono, K. Koshino, Y. Tabuchi, A. Noguchi, and Y. Nakamura, Quantum non-demolition detection of an itinerant microwave photon, Nat. Phys. 14, 546 (2018).

[54] S. Parkins and T. Aoki, Microtoroidal cavity QED with fiber overcoupling and strong atom-field coupling: A single-atom quantum switch for coherent light fields, Phys. Rev. A 90, 053822 (2014).

[55] H. Mabuchi and A. C. Doherty, Cavity quantum electrodynamics: Coherence in context, Science 298, 1372 (2002).

[56] D. Maxwell, D. J. Szwer, D. Paredes-Barato, H. Busche, J. D. Pritchard, A. Gauguet, K. J. Weatherill, M. P. A. Jones, and C. S. Adams, Storage and Control of Optical Photons Using Rydberg Polaritons, Phys. Rev. Lett. 110, 103001 (2013).

[57] M. Fleischhauer and M. D. Lukin, Quantum memory for photons: Dark-state polaritons, Phys. Rev. A 65, 022314 (2002).

[58] M. D. Lukin, Colloquium: Trapping and manipulating photon states in atomic ensembles, Rev. Mod. Phys. 75, 457 (2003).

[59] A. V. Gorshkov, A. André, M. D. Lukin, and A. S. Sørensen, Photon storage in $\Lambda$-type optically dense atomic media. II. Free-space model, Phys. Rev. A 76, 033805 (2007).

[60] Y. Wang, J. Li, S. Zhang, K. Su, Y. Zhou, K. Liao, S. Du, H. Yan, and S.-L. Zhu, Efficient quantum memory for single-photon polarization qubits, Nat. Photonics 13, 346 (2019).

[61] A. Reiserer, N. Kalb, G. Rempe, and S. Ritter, A quantum gate between a flying optical photon and a single trapped atom, Nature (London) 508, 237 (2014).

[62] L.-M. Duan and H. J. Kimble, Scalable Photonic Quantum Computation through Cavity-Assisted Interactions, Phys. Rev. Lett. 92, 127902 (2004).

[63] B. Wang and L.-M. Duan, Engineering superpositions of coherent states in coherent optical pulses through cavity-assisted interaction, Phys. Rev. A 72, 022320 (2005).

[64] X.-M. Lin, Z.-W. Zhou, M.-Y. Ye, Y.-F. Xiao, and G.-C. Guo, One-step implementation of a multiqubit controlled-phase-flip gate, Phys. Rev. A 73, 012323 (2006).

[65] S. Schmidt-Eberle, T. Stolz, G. Rempe, and S. Dürr, Dark-time decay of the retrieval efficiency of light stored as a Rydberg excitation in a noninteracting ultracold gas, Phys. Rev. A 101, 013421 (2020).

[66] Y. O. Dudin, L. Li, and A. Kuzmich, Light storage on the time scale of a minute, Phys. Rev. A 87, 031801(R) (2013).

[67] I. I. Beterov, I. I. Ryabtsev, D. B. Tretyakov, and V. M. Entin, Quasiclassical calculations of blackbody-radiation-induced depopulation rates and effective lifetimes of Rydberg $n S, n P$, and $n D$ alkali-metal atoms with $n \leqslant 80$, Phys. Rev. A 79, 052504 (2009).

[68] D. Petrosyan, G. Bensky, G. Kurizki, I. Mazets, J. Majer, and J. Schmiedmayer, Reversible state transfer between superconducting qubits and atomic ensembles, Phys. Rev. A 79, 040304(R) (2009).

[69] C. M. Caves, Quantum limits on noise in linear amplifiers, Phys. Rev. D 26, 1817 (1982). 
[70] Actually, the distortion of the output state due to unbalanced $C_{1,2}^{o L / R}$ can be solved by adding a unitary rotation. However, such a rotation will be different for different input states, while tuning the loss to balance $C_{1,2}^{o L / R}$ is robust to different input states.

[71] Jozsa and Richard, Fidelity for mixed quantum states, J. Mod. Opt. 41, 2315 (1994).

[72] A. Megrant, C. Neill, R. Barends, B. Chiaro, Y. Chen, L. Feigl, J. Kelly, E. Lucero, M. Mariantoni, P. O’Malley, D. Sank, A. Vainsencher, J. Wenner, T. White, Y. Yin, J. Zhao, C.
Palmstrom, J. Martinis, and A. Cleland, Planar superconducting resonators with internal quality factors above one million, Appl. Phys. Lett. 100, 113510 (2012).

[73] The material here is a re-derivation of the classic FleischauerLukin paper [57] using our own approach.

[74] This is a direct result of the fluctuation-dissipation theorem.

[75] Y. He, L. Ji, Y. Wang, L. Qiu, J. Zhao, Y. Ma, X. Huang, S. Wu, and D. E. Chang, Geometric Control of Collective Spontaneous Emission, Phys. Rev. Lett. 125, 213602 (2020). 